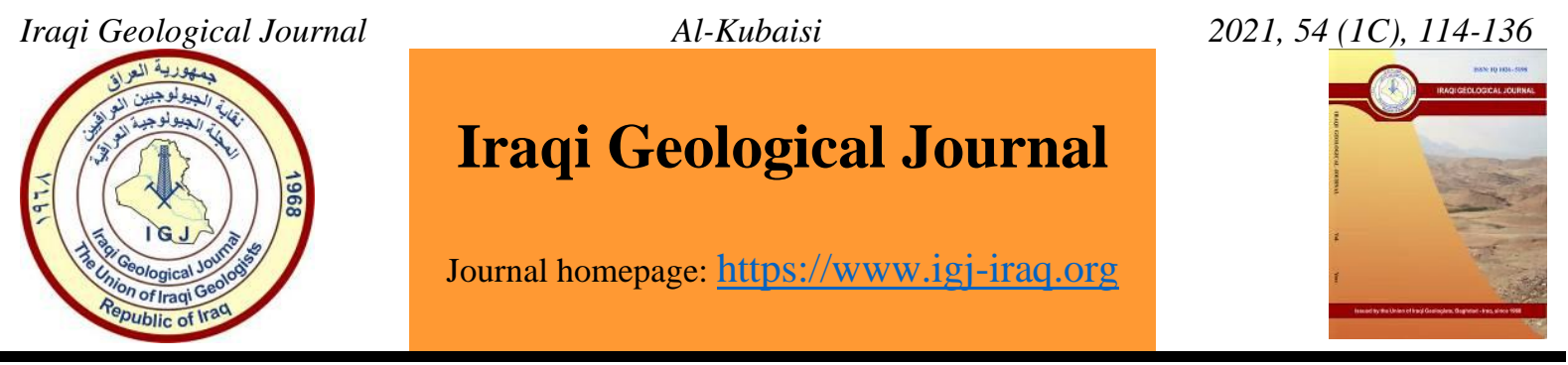

\title{
An Integrative Modeling Approach to Estimating Volume of Generated Oil from Lower Cretaceous Sulaiy Formation in Southern Iraq
}

\author{
Basim F. Al-kubaisi ${ }^{1}{ }^{*}$, Mohammed A. Al-Nuaimi ${ }^{1}$ and Amer S. Al-jibouri ${ }^{1}$ \\ Department of Applied Geology, College of Science, University of Anbar, Anbar, Iraq \\ *Correspondence: basimalkubaisi@uoanbar.edu.iq \\ Received: 24 July 2020; Accepted: 18 January 2021; Published :31 March 2021
}

\begin{abstract}
Thermal maturity and petroleum generation capacity of the source rocks of Lower Cretaceous Sulaiy Formation were studied and evaluated via modeling the thermal maturation of ten wells in this study by using the Arrhenius equation for chemical reactions or (time-temperature index of thermal maturation). A thermal maturation map of the Sulaiy Formation shows that it a high maturity level increasing eastward; also, the geochemical analysis indicated suitable organic content in quantity and quality to generate hydrocarbons. The onset of oil migration from the Sulaiy Formation is calculated by this study to be at the end of Lower Eocene time (53 million years ago), and as it is a late hydrocarbon generation and migration; thus indicating a mainly vertical migration paths. According to quantitative estimation, the oil generated and migrated vertically from the Sulaiy Formation towards the Lower Cretaceous reservoirs in southern Iraq estimated at $42.6 \times 10^{9} \mathrm{bbl}$. This study elevated the status of the Sulaiy Formation to a major source of oil tapped in the Lower Cetaceous reservoirs of southern Iraq.
\end{abstract}

Keywords: Source Rock; Arrhenius Equation; Sulaiy Formation; Iraq

\section{Introduction}

The process of identifying oil generating rocks is considered within any zone is a basic and effective tool in the study of the hydrocarbon potential of that zone and is an important factor in reducing the risk factor in exploratory process. The Sulaiy Formation considered to be the most important source rock in southern Iraq, This Formation of Tithonian - Berriasian age consists of detrital, oolitic limestone and hard crystalline limestone interbedded with shale (Jassim and Buday, 2006), overlain conformably by Ratawi or Yamama formations and underlain unconformable by the Gotnia Formation. The black shales between the limestone beds can be considered as a good source for hydrocarbons generation because the shales are rich in organic matters and have reached the level of maturation zone (Mamaseni, 2020). The thickness of it ranging between $100-400 \mathrm{~m}$ and decreasing until wedge out to the west. Previously, many researchers have developed a digital scale of thermal maturation by adopting mathematical models that combine the thermal and temporal history of a sedimentary horizon. (Karweil, 1956; Waples, 1980; Royden et al., 1980). Determining the thermal maturation level and oil generation prospects depends on one of the following principles: The rate of chemicals reaction doubles by $10^{\circ} \mathrm{C}$ for each heat increment (Waples, 1980). The second principles, which was adopted in this study, is based

DOI: $\underline{10.46717 / i g j .54 .1 C .9 M s-2021-03-29}$ 
on the Arrhenius equation for chemical reactions, which provides for logarithmic increases for kerogen degradation. This equation requires the identification of two important factors, which are the $\mathrm{E}$ and $\mathrm{A}$ represents activation energy and pre-exponential factor, respectively (Snowdon, 1979). Hatif et al. (2020) mentioned that kerogen types of the formation are mostly type II (algal marine). The rate of convertible kerogen is exponentially proportional to time depending on the principle of Arrhenius equation (Connan, 1974). The chemical reaction pathway of the three main types of kerogen (I, II, III) was determined for the purpose of laying the practical foundations for the thermal maturation of organic matter. These types of kerogen have been classified on the atomic ratios $(\mathrm{H} / \mathrm{C}$ and $\mathrm{O} / \mathrm{C})$ extracted from the analysis of the main elements of kerogen using the C, H, N analyzer (Tissot and Espitalie, 1975; Tissot and Welte, 1978). The scale of organic maturation used in Arrhenius equation depends on increasing of activation energy (E) with increased temperature. This scale equals to the adopted scale in the Lopatin equation that is containing a double reaction rate for each temperature increase of $10^{\circ} \mathrm{C}$ (Wood, 1988). Two widely diffused methods, one based on the Lopatin principle (Lopatin, 1971) and the other on the basis of Arrhenius equation, showed the basic contradictions between the results of the different methods (Wood, 1988).

\section{Study Area}

Ten exploratory wells were selected that penetrated the Sulaiy Formation in southern Iraq, according to the availability of their stratigraphic and geochemical data. They are wells ( $\mathrm{Zb}-47, \mathrm{Mj}-12$, Lu-12, KI-1, Ak-1, Ub-1, Sw-1, Sa-1, Gh-1, and Si-1). The provided a coverage of almost all of southern Iraq between longitude $43^{\circ}-48^{\circ}$, and latitude $29^{\circ}-32^{\circ}$, it is specified by the following coordinates in the UTM system / E: 300 - 800, N: 3200 - 3500. (Fig .1). The study area has a geological and tectonic history, which has been related to a series of tectonic movements, which had an obvious effect in shaping the sedimentation framework for this region. The eastern part of the region is characterized by its economic importance, as the largest oil fields in Iraq are located.

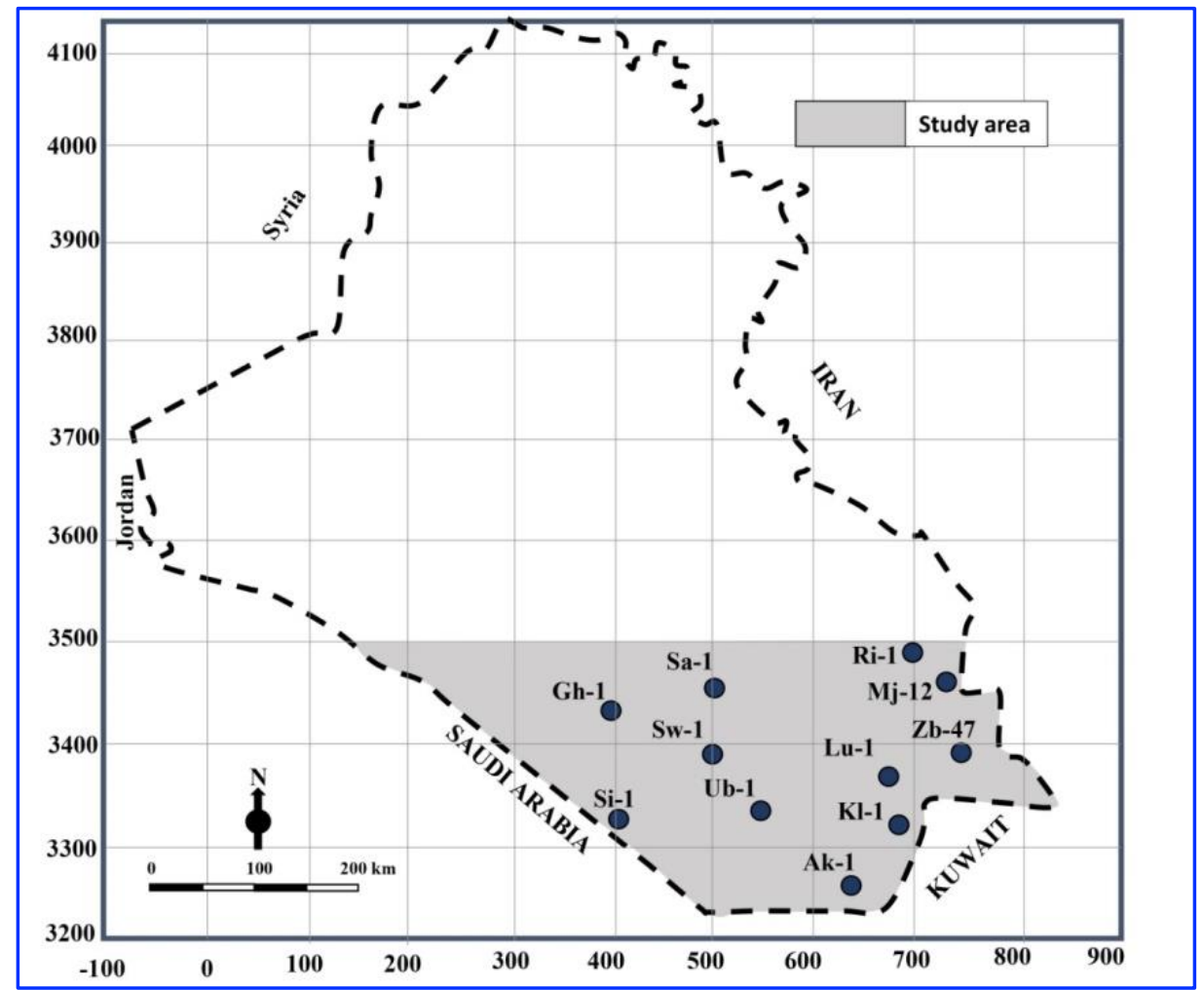

Fig. 1. Location map for the study area, and locations of the selected wells 


\section{Materials and Methods}

The primary inputs of the Arrhenius equation (the thickness of stratigraphic units and the start and end times of sedimentation of each of these units for all stratigraphic columns) (Table 1-10). The unconformities were also identified and calculate the eroded thickness within the sequence. The geothermal gradient of these wells is determined by Ibrahim, 1984 and Japan National Oil Corporation's report at the Iraqi National Oil Company's library (1988). Above extrapolated values were used in the application of Arrhenius model for the purpose of determining the thermal maturation zone of the base of the Sulaiy Formation. Finally, the results of the geochemical analyzes were treated as essential inputs within the Espitalie model to calculated the amount of oil generated within the Sulaiy Formation.

\subsection{Arrhenius Model for Thermal Maturation}

The factors of time and temperature are playing an important role in organic metamorphism into hydrocarbons, as the key factors in the kerogen breakdown are time and temperature (Allen and Allen, 2005). For the purpose of determining the thermal maturation level of a specific sequence and then determines the oil potential for that sequence. The geochemical indices have been widely used in this field. However, these indices are only determined the present situation of thermal maturation. Since, they do not reflect changes in the organic matter in the source rocks through the history of their thermal maturation (Ejedawe et al, 1984). Wood (1988) suggested a mathematical model for thermal maturation based mainly on the equation of kinetic chemical reactions known as Arrhenius equation which represents the logarithmic dependence of chemical reaction on heat.

The published analysis results indicate values of activation energy ranging from 100 to 350 $\mathrm{KJ} / \mathrm{mol}$, and pre-exponential factors values ranging between (40-80/m.y.) (Lewan, 1985). The values approved for both $\mathrm{E}, \mathrm{A}$ in this study are $(\mathrm{E}=218 \mathrm{kj} / \mathrm{mol}),\left(\mathrm{A}=5.45 \mathrm{X} 10^{26} / \mathrm{m} . \mathrm{y}\right.$.). These are the approved values for type II Kerogen (Snowdon, 1979).In order for crude oil to be generated within the source rock, many chemical reactions must take place, the early reactions would generate a small fraction of convertible kerogen, which is unable to migrate or be expelled out of the source rock, also, the late products of the transformation of organic matter are also unimportant in the generation of oil due to its behavior to generating gases, Therefore, the quantities generated at the peak of oil generation window are the most important and have the ability to migrate (Tissot,1984). Chemical kinetics is the most ideal approach to evaluate the generation and maturation of hydrocarbon components, molecular biomarkers and coal macerals. Amid the recent study, a set of chemical kinetics is chosen to describe the generation, quality and quantity of hydrocarbons (Hantschel and Kauerauf, 2009). The kinetic parameters of the main assumed types of kerogen( I, II, III )have been used on the basis of the mass fragmentation for each contribute to the overall petroleum potential for each type of kerogen(Tissot and Espitalie, 1975). These three values are obtained of the mean of kerogen types on the other hand give a linear trend between activation energy and pre-exponential factor. This relationship shows a beginning of the temperature limits in which transformation of the reactive parts of different types of kinetic reactions.

\subsection{Arrhenius's Mathematical Expression}

The mathematical expression of the thermal maturation model based on Arrhenius equation is:

$$
K_{A R R}=\mathrm{A} \exp (-\mathrm{E} / \mathrm{RT})
$$

To evaluate the (TTI) for any thermal horizon, the integral of the heat range is multiplied by the time elapsed with the heat interval. The $\operatorname{TTI}_{A R R}\left(\mathrm{t}_{\mathrm{n}}\right.$ to $\left.\mathrm{t}_{\mathrm{n}+1}\right)$ it can be expressed as:

$$
T T I_{A R R}\left(\mathrm{t}_{\mathrm{n}} \text { to } \mathrm{t}_{\mathrm{n}+1}\right)=\frac{A}{q_{n}} \int_{T_{n}}^{T_{n+1}} \exp (-\mathrm{E} / \mathrm{RT}) d T
$$


Gorbachev (1975) provided an integral analysis of constant temperature with time:

$$
\operatorname{TTI}_{A R R}\left(T_{n}^{t_{n} \text { to } t_{n+1}}=0 k=\frac{A}{q_{n}} \frac{R T_{n+1}^{2}}{E+2 R T_{n+1}} \exp \left(-\mathrm{E} / \mathrm{R} T_{n+1}\right)\right.
$$

When temperature changes are constant, the time-temperature indices can be determined for a specific temperature level:

$$
\operatorname{TTI}_{\mathrm{ARR}}\left(T_{n \neq T_{n+1}}^{t_{n} \text { to } t_{n+1}}\right)=\frac{A}{q_{n}}\left\{\frac{R T_{n+1}^{2}}{E+2 R T_{n+1}} \exp \left(-\mathrm{E} / \mathrm{R} T_{n+1}\right)-\frac{R T_{n}^{2}}{E+2 R T_{n}} \exp \left(-\mathrm{E} / \mathrm{R} T_{n}\right)\right.
$$

Some burial history curves have long time periods at a constant temperature with low rate of subsidence or uplift. To account for those time periods at a constant temperature on a given burial history curve a $\mathrm{TTI}_{\mathrm{ARR}}$ value can be calculated:

$$
\operatorname{TTI}_{\mathrm{ARR}}\left(T_{n}^{t_{n} t o t_{n+1}}\right)=\left(\mathrm{t}_{n+1}-\mathrm{t}_{\mathrm{n}}\right) \mathrm{A} \exp \left(-\mathrm{E} / \mathrm{R} T_{n}\right)
$$

Assuming that the thermal history of sediments are separated periods, and for a constant increase or decrease in temperature, the calculation of cumulative maturity indices can be expressed as:

$$
\begin{gathered}
\sum T T I_{A R R}=\left\{\sum_{n=1}^{n=m} \frac{A}{q_{n}}\left(\frac{R T_{n+1}^{2}}{E+2 R T_{n+1}} \exp \left(-\mathrm{E} / \mathrm{R} T_{n+1}\right)-\frac{R T_{n}^{2}}{E+2 R T_{n}} \exp \left(-\mathrm{E} / \mathrm{R} T_{n}\right)\right\}+\{\right. \\
\sum_{ \pm=1}^{n=m}\left(t_{n+1}-t_{n}\right) \mathrm{A} \exp \left(-\mathrm{E} / \mathrm{R} T_{n}\right\}
\end{gathered}
$$

The heating rate for a specific time interval, can be determined from burial history curve, here the geothermal gradient must be calculated (Ejedawe \& Coker, 1984).A map of the geothermal gradient of southern Iraq has been adopted (Ibrahim, 1984) and (I.N.O.C., 1988) in this study to determine the geothermal gradient for the study area. Finally, the main target for Arrhenius method is to determine the thermal maturation zone of the studied sequence, meaning determine the oil generative window $(\mathrm{O} G$ W). Wood (1988) suggested the following equation to determine the oil generation window:

$$
\mathrm{X}=\exp \left(-\sum T T I_{A R R}\right)
$$

$\mathrm{X}$ is directly related to $\log _{10} \sum T T I_{A R R}$ values, when this value is less than -4 then the value of $\mathrm{X}$ are basically equal to 1 , this, means that the organic matter have not yet entered within the thermal maturation zone. When the $\log _{10} \sum T T I_{A R R}$ values become more than +1 then the value of $\mathrm{X}$ equal to 0 meaning that the convertible of kerogen into hydrocarbons has completely occurred. Accordingly, the oil-generative window based on Arrhenius model is determined at the values of $\log _{10} \sum T T I_{A R R}$, which are between $+1,-4$

\section{3. $\log _{10} \sum T T I_{l o p}-\log _{10} \sum T T I_{A R R}-R_{o}$ Relationships}

Waples (1982) suggested a table for the limits of the generation zone in the Lopatin method, where the beginning and the end of the thermal maturation zone is determined at the values of TTI $(15,160)$. The peak of thermal maturity is determined when the value of 75 It is possible to convert the TTI values to other maturation indices such as vitrinite reflectance $\left(R_{o}\right)$. The cubic regression equation 
for empirical correlation betweenLog $\log _{10} R_{o}$ andLog $\log _{10} \sum T T I_{\text {lop }}$ can be obtained for the range $R_{o} 0.3-5.0$ $\%$ and $\log _{10} \sum T T I_{l o p}-2.0$ to 4.9 :

$$
\log _{10} R_{o} \%=-0.006\left(\log _{10} \sum T T I_{\text {lop }}\right)^{3}+0.042\left(\log _{10} \sum T T I_{\text {lop }}\right)^{2}+0.162\left(\log _{10} \sum T T I_{\text {lop }}\right)-0.397
$$

Like Waples determination of $R_{o}$ - TTI relationship, Wood formulated a mathematical equation to calculate $R_{o}$ values by thermal maturity factors:

$$
\log _{10} R_{o} \%=-0.0001\left(\log _{10} \sum T T I_{A R R}\right)^{3}+0.003\left(\log _{10} \sum T T I_{A R R}\right)^{2}+0.109\left(\log _{10} \sum T T I_{A R R}+0.106\right.
$$

The calculated values of $\log _{10} \sum T T I_{A R R}, R_{o}$ and the remaining of convertible kerogen have been linked to the graphic relationship in order to determine the oil generating window and the peak of the oil generation (Fig. 2).There is a temperature level between $\left(10-30^{\circ} \mathrm{C}\right)$ below this level of temperature no maturity of vitrinite occurred and this limit of vitrinite maturity corresponds to the value of a light reflection of the vitrine around (0.35-0.40\%) (Learche et al, 1984). Therefore the calculated $\sum$ TTIvalues computed according to Arrhenius's model should be approximated or can be compared well with the vitrinite reflectance.

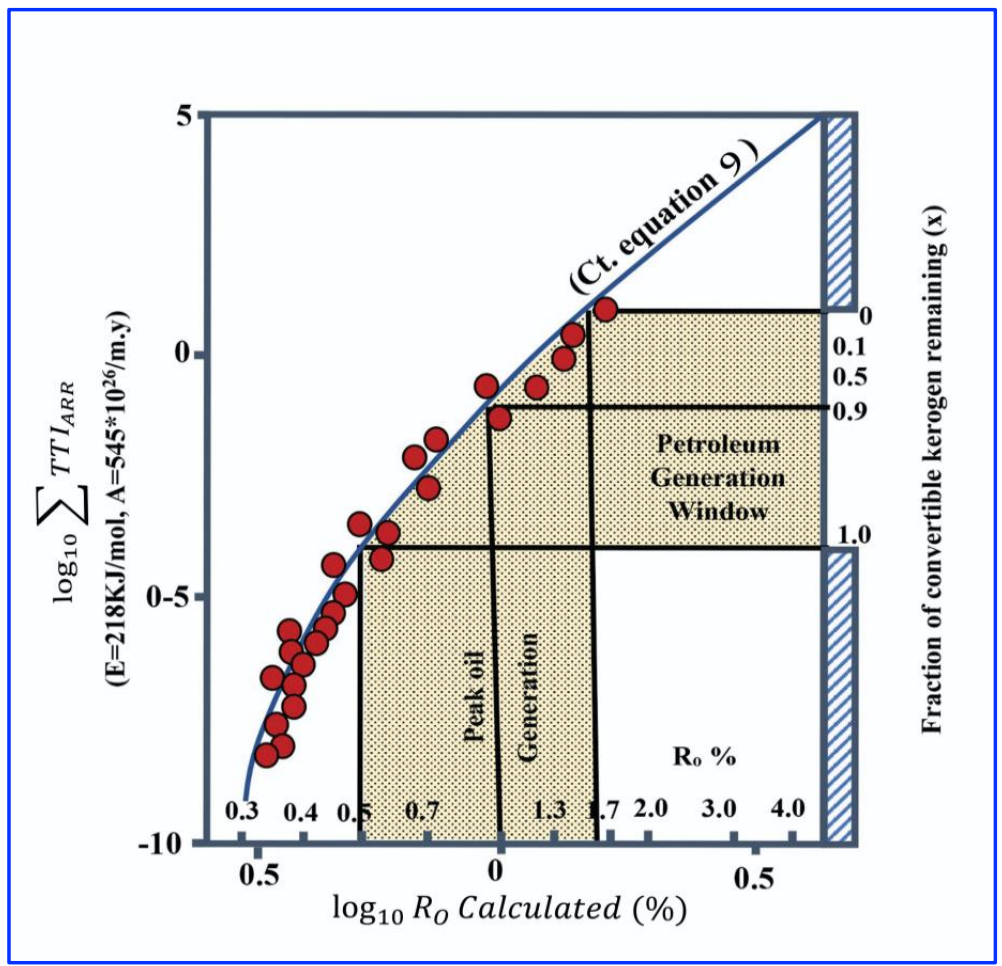

Fig .2. $\log _{10} \sum T T I_{A R R}, \log _{10} R_{o}$, Fraction of conv. Kerogen remaining (X) relationship (After: Wood,1988)

The value $\left(\sum T T I=0\right)$ corresponds to the vitrinite reflectance value equal to $(0.35 \%)$ it is through the identification of $\left(\sum T T I=0\right)$ (Table-1).We actually find it falling at a value $\left(\log _{10} \sum T T I_{A R R}=\right.$ $-6.59)$ which is corresponding to $\left(R_{o}\right)$ value of $(0.35)$.

\subsection{Evaluation of Thermal Maturity}

The thermal maturation coefficient of ten boreholes distributed across the southern parts of Iraq is (Zb-47, Mj-12, Lu-12, KI-1, Ak-1, Ub-1, Sw-1, Sa-1, Gh-1, and Si-1) (Tables 1-10). The thermal maturation values calculated according to Arrhenius equation that has been used for Iso-TTI mapping of Sulaiy Formation (Fig.10). It is very likely that Sulaiy Formation has generated an important part of 
the oil migrating vertically towards Lower Cretaceous reservoir. The analysis show that the oil samples represented one family, non-degraded, marine and non-waxy deposits were derived from carbonate and marl source rocks deposited in anoxic marine environment (Al-Mimar, 2014). On the other hand, the units of Jurassic are unlikely to have generated any part of that oil because of the impossibility of a vertical migration of oil through the contact between Upper Jurassic and Lower Cretaceous (Gotnia evaporite). Therefore, all the source rocks pre-Gotnia are excluded from the present estimates and this is supported by (Ibrahim, 1983). The thermal mature part of the Sulaiy Formation covers relatively a wide area where, the values of thermal maturation are increased eastward with high rates of maturity, whereas the heat flow in range $\left(27-70 \mathrm{w} / \mathrm{m}^{2}\right)$ which influence in the thermal maturity (Handhal and Mahdi, 2016). Generally, the lower- middle Cretaceous succession lies of the mid-oil window condition (Al-Ameri et al. 2011). And especially the expulsion in the Sulaiy Formation in Ri-1 well is higher than all formations in all of the studied wells in southern part of Iraq (Al- Zaidi, 2019) (Fig. 3). The thermal maturity indices at well $(\mathrm{Mj}-12)$ (Table-1) indicated that it entered the maturation zone at (52 million years ago) during the end of Lower Eocene. In addition to the appropriate thermal conditions for oil generation, the lower part of the formation contains a high quantity of total organic matter up to $7.7 \%$ in some depth (OEC, 1987). These matters mostly marine with high potential in production of hydrocarbons. The values of $\mathrm{H} / \mathrm{C}$ versus $\mathrm{O} / \mathrm{C}$, and $\mathrm{HI}$ versus $\mathrm{T}_{\max }$ extracted from above OEC report indicate the dominance of type II kerogen, and sometime tends towards the type III (Figs 4 and 5). Also, the values of $\mathrm{TOC}_{\mathrm{o}} \%$ versus $\mathrm{PP}_{\mathrm{o}}$ and $\mathrm{TOC}_{\mathrm{o}} \%$ versus depth calculated according to the equation (17) and shown in Figs. 6 and 7 represented high content of organic matter and a high petroleum potential to hydrocarbon productions. The initial total organic carbon $\left(\mathrm{TOC}_{\mathrm{o}}\right.$ ) was determined by projecting the $\mathrm{H} / \mathrm{C}$ ratio on the Baskin diagram (Baskin, 1997) which determines the percentage of organic carbon lost that is added to the remaining TOC to calculate $\mathrm{TOC}_{\mathrm{o}}$. The oil generated zone was also determined by the projecting of the calculated values $R_{o}$ versus depth (Fig. 8). The projection of the calculated and observed TR values versus depth is used to determine the main zone of oil generation within the Sulaiy Formation which is determined at depth (4480-4510 m.) (Fig. 9).Therefore, according to the above results we can consider the rocks of the Sulaiy Formation as a well-supplied source of oil for southern Iraq reservoir and some surrounding areas. Stoneley (1987) emphasized that appropriateness of the formation rocks locally in oil generation in northern part of the Arabian Gulf (South-East of Iraq and Northern Kuwait).

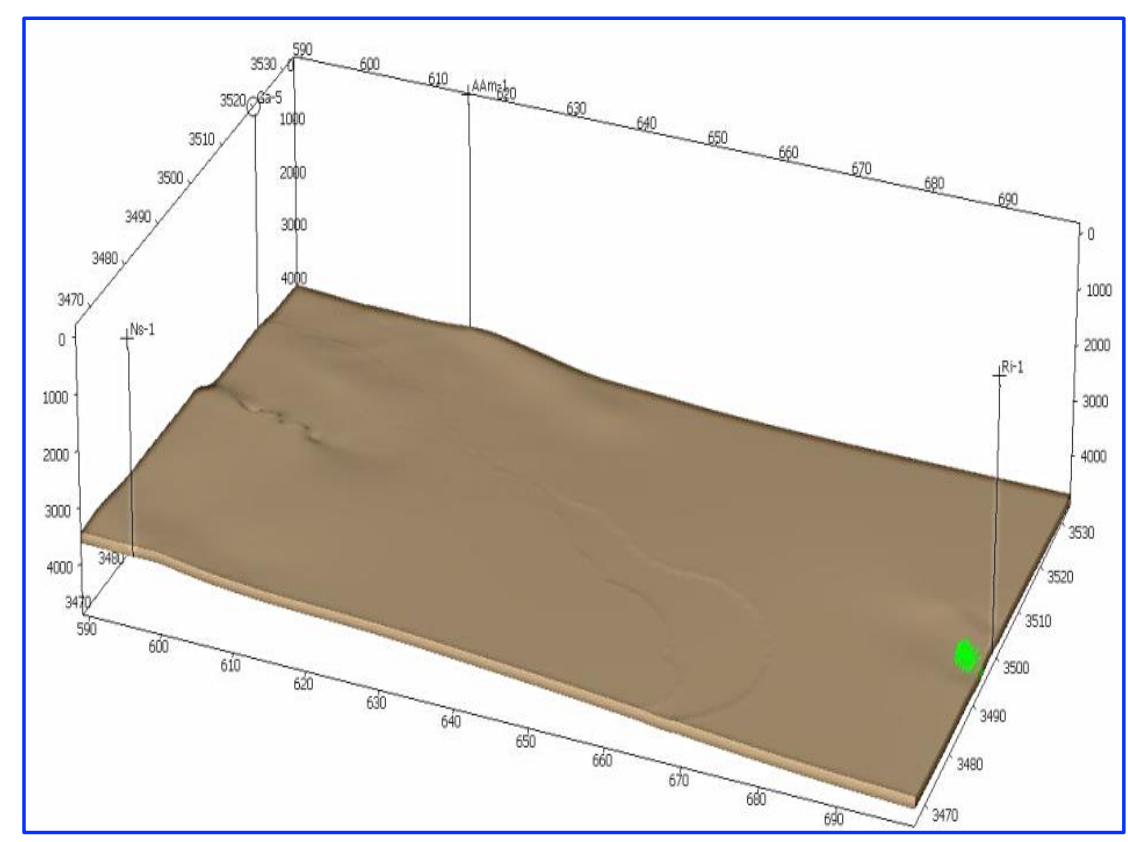

Fig. 3. Expulsion of oil for Sulaiy Formation in Ri-1 well, after (Al-Zaidi, 2019) 
Table.1 Calculation and results of thermal maturity values of well $\mathrm{Mj}-12$

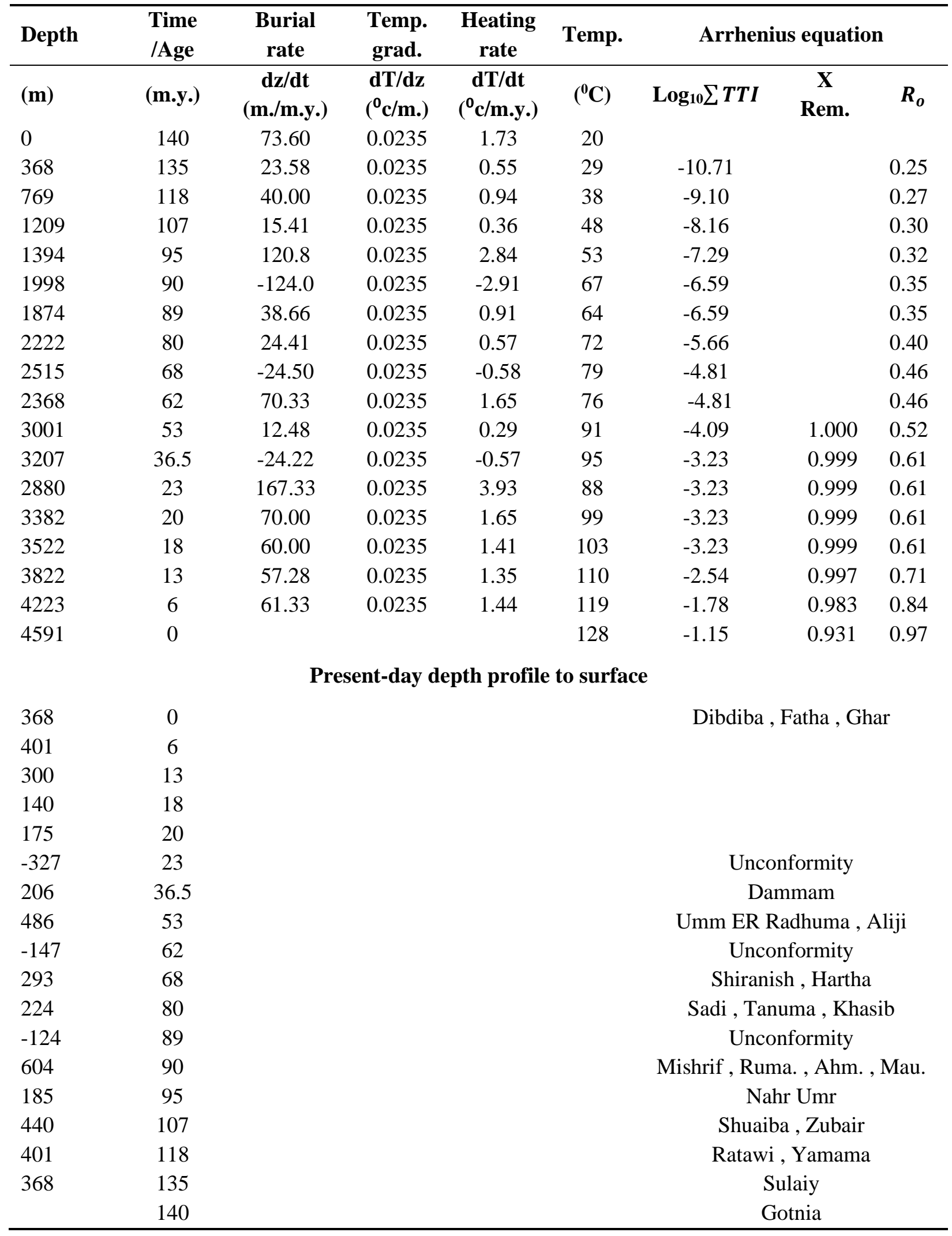


Table 2. Calculations and results of thermal maturity values of well $\mathrm{Zb}-47$

\begin{tabular}{|c|c|c|c|c|c|c|c|c|}
\hline Depth & $\begin{array}{l}\text { Time/ } \\
\text { Age }\end{array}$ & $\begin{array}{l}\text { Burial } \\
\text { rate }\end{array}$ & $\begin{array}{l}\text { Temp. } \\
\text { grad. }\end{array}$ & $\begin{array}{c}\text { Heating } \\
\text { rate }\end{array}$ & Temp. & \multicolumn{3}{|c|}{ Arrhenius equation } \\
\hline (m) & $(\mathbf{m . y .})$ & $\begin{array}{c}\text { dz/dt } \\
(\mathrm{m} . / \mathrm{m} . \mathbf{y} .)\end{array}$ & $\begin{array}{l}\text { dT/dz } \\
\left({ }^{0} \mathrm{c} / \mathrm{m} .\right)\end{array}$ & $\begin{array}{c}\text { dT/dt } \\
\left({ }^{0} \mathrm{c} / \mathrm{m} . \mathrm{y} .\right)\end{array}$ & $\left({ }^{0} \mathrm{C}\right)$ & $\log _{10} \sum T T I$ & $\begin{array}{c}X \\
\text { Rem. }\end{array}$ & $\boldsymbol{R}_{o}$ \\
\hline 0 & 140 & 50.40 & 0.022 & 1.11 & 20 & & & \\
\hline 252 & 135 & 31.35 & 0.022 & 0.69 & 26 & -10.94 & & 0.25 \\
\hline 785 & 118 & 39.45 & 0.022 & 0.87 & 37 & -9.30 & & 0.27 \\
\hline 1219 & 107 & 27.66 & 0.022 & 0.61 & 47 & -8.20 & & 0.30 \\
\hline 1551 & 95 & 115.6 & 0.022 & 2.54 & 54 & -7.35 & & 0.32 \\
\hline 2129 & 90 & -84.00 & 0.022 & -1.8 & 67 & -6.53 & & 0.36 \\
\hline 2045 & 89 & 56.22 & 0.022 & 1.24 & 65 & -6.53 & & 0.36 \\
\hline 2551 & 80 & 34.00 & 0.022 & 0.75 & 76 & -6.53 & & 0.36 \\
\hline 2959 & 68 & 37.00 & 0.022 & 0.81 & 85 & -4.34 & & 0.49 \\
\hline 3070 & 65 & -60.33 & 0.022 & -1.33 & 88 & -4.34 & & 0.49 \\
\hline 2889 & 62 & 134.0 & 0.022 & 2.95 & 81 & -4.34 & & 0.49 \\
\hline 3291 & 59 & 38.16 & 0.022 & 0.84 & 92 & -4.33 & 1.000 & 0.50 \\
\hline 3520 & 53 & 22.77 & 0.022 & 0.50 & 97 & -3.46 & 0.999 & 0.59 \\
\hline 3725 & 44 & 21.33 & 0.022 & 0.47 & 102 & -2.82 & 0.998 & 0.67 \\
\hline 3885 & 36.5 & -26.37 & 0.022 & -0.58 & 105 & -2.82 & 0.998 & 0.67 \\
\hline 3529 & 23 & 52.07 & 0.022 & 1.15 & 98 & -2.82 & 0.998 & 0.67 \\
\hline 4258 & 9 & 28.00 & 0.022 & 0.62 & 114 & -2.03 & 0.991 & 0.79 \\
\hline 4510 & 0 & & & & 119 & -1.58 & 0.974 & 0.87 \\
\hline \multicolumn{9}{|c|}{ Present-day depth profile to surface } \\
\hline 252 & 0 & & & & & \multicolumn{3}{|c|}{ Dibdiba., Fatha, Ghar } \\
\hline 373 & 9 & & & & & & & \\
\hline-356 & 23 & & & & & \multicolumn{3}{|c|}{ Unconformity } \\
\hline 160 & 36.5 & & & & & \multicolumn{3}{|c|}{ Dammam, Rus } \\
\hline 205 & 44 & & & & & & & \\
\hline 229 & 53 & & & & & \multicolumn{3}{|c|}{ Umm ER Radhuma } \\
\hline 221 & 54 & & & & & & & \\
\hline-181 & 62 & & & & & \multicolumn{3}{|c|}{ Unconformity } \\
\hline 111 & 65 & & & & & \multicolumn{3}{|c|}{ Tayarat, Shiranish , Hartha } \\
\hline 408 & 68 & & & & & & & \\
\hline 422 & 80 & & & & & \multicolumn{3}{|c|}{ Sadi, Tanuma., Khasib } \\
\hline-84 & 89 & & & & & \multicolumn{3}{|c|}{ Unconformity } \\
\hline 578 & 90 & & & & & \multicolumn{3}{|c|}{ Mishrif, Ruma., Ahm. , Mau. } \\
\hline 332 & 95 & & & & & \multicolumn{3}{|c|}{ Nahr Umr } \\
\hline 434 & 107 & & & & & \multicolumn{3}{|c|}{ Shuaiba, Zubair } \\
\hline 533 & 118 & & & & & \multicolumn{3}{|c|}{ Ratawi, Yamama } \\
\hline 252 & 135 & & & & & \multicolumn{3}{|c|}{ Sulaiy } \\
\hline & 140 & & & & & \multicolumn{3}{|c|}{ Gotnia } \\
\hline
\end{tabular}


Table 3. Calculations and results of thermal maturity values of well Lu-12

\begin{tabular}{|c|c|c|c|c|c|c|c|c|}
\hline Depth & $\begin{array}{l}\text { Time } \\
\text { /Age }\end{array}$ & $\begin{array}{l}\text { Burial } \\
\text { rate }\end{array}$ & $\begin{array}{l}\text { Temp. } \\
\text { grad. }\end{array}$ & $\begin{array}{c}\text { Heating } \\
\text { rate }\end{array}$ & Temp. & \multicolumn{3}{|c|}{ Arrhenius equation } \\
\hline (m) & (m.y.) & $\begin{array}{c}\text { dz/dt } \\
(\mathbf{m} . / \mathrm{m} . \mathbf{y} .)\end{array}$ & $\begin{array}{l}\mathrm{dT} / \mathrm{dz} \\
\left({ }^{0} \mathrm{c} / \mathrm{m} .\right)\end{array}$ & $\begin{array}{c}\text { dT/dt } \\
\left({ }^{0} \mathrm{c} / \mathrm{m} . \mathrm{y} .\right)\end{array}$ & $\left({ }^{0} \mathrm{C}\right)$ & $\begin{array}{r}\log _{10} \\
\sum T T I\end{array}$ & X Rem. & $\boldsymbol{R}_{\boldsymbol{o}}$ \\
\hline 0 & 140 & 50.00 & 0.0188 & 0.94 & 20 & & & \\
\hline 250 & 135 & 32.05 & 0.0188 & 0.60 & 25 & -11.04 & & 0.25 \\
\hline 795 & 118 & 45.63 & 0.0188 & 0.86 & 35 & -9.49 & & 0.27 \\
\hline 1297 & 107 & 19.33 & 0.0188 & 0.36 & 44 & -8.58 & & 0.28 \\
\hline 1529 & 95 & 73.80 & 0.0188 & 1.39 & 49 & -7.73 & & 0.31 \\
\hline 1925 & 90 & -63.00 & 0.0188 & -1.18 & 56 & -7.49 & & 0.32 \\
\hline 1862 & 89 & 44.33 & 0.0188 & 0.83 & 55 & -7.49 & & 0.32 \\
\hline 2261 & 80 & 46.20 & 0.0188 & 0.87 & 63 & -6.52 & & 0.36 \\
\hline 2492 & 75 & 42.50 & 0.0188 & 0.80 & 67 & -6.28 & & 0.37 \\
\hline 2917 & 65 & -76.66 & 0.0188 & -1.44 & 75 & -5.31 & & 0.42 \\
\hline 2687 & 62 & 107.8 & 0.0188 & 2.03 & 71 & -5.31 & & 0.42 \\
\hline 3226 & 57 & 37.25 & 0.0188 & 0.70 & 81 & -5.13 & & 0.44 \\
\hline 3375 & 53 & 21.12 & 0.0188 & 0.45 & 83 & -4.84 & & 0.46 \\
\hline 3773 & 36.5 & -26.00 & 0.0188 & -0.49 & 91 & -3.60 & 1.000 & 0.57 \\
\hline 3422 & 23 & 26.04 & 0.0188 & 0.49 & 84 & -3.50 & 0.999 & 0.58 \\
\hline 4021 & 0 & & & & 96 & -3.15 & 0.998 & 0.62 \\
\hline \multicolumn{9}{|c|}{ Present-day depth profile to surface } \\
\hline 248 & 0 & & & & & \multicolumn{3}{|c|}{ Dibdiba, Fatha, Ghar } \\
\hline-351 & 23 & & & & & \multicolumn{3}{|c|}{ Unconformity } \\
\hline 398 & 36.5 & & & & & \multicolumn{3}{|c|}{ Dammam, Rus } \\
\hline 149 & 53 & & & & & \multicolumn{3}{|c|}{ Umm ER Radhuma } \\
\hline 309 & 57 & & & & & & & \\
\hline-230 & 62 & & & & & \multicolumn{3}{|c|}{ Unconformity } \\
\hline 425 & 65 & & & & & \multicolumn{3}{|c|}{ Tayarat, Shiranish, Hartha } \\
\hline 231 & 75 & & & & & & & \\
\hline 336 & 80 & & & & & \multicolumn{3}{|c|}{ Sadi , Tanuma, Khasib } \\
\hline-63 & 89 & & & & & \multicolumn{3}{|c|}{ Unconformity } \\
\hline 396 & 90 & & & & & \multicolumn{3}{|c|}{ Mishrif, Ruma. , Ahm., Mau. } \\
\hline 232 & 95 & & & & & \multicolumn{3}{|c|}{ Nahr Umr } \\
\hline 502 & 107 & & & & & \multicolumn{3}{|c|}{ Shuaiba , Zubair } \\
\hline 545 & 118 & & & & & \multicolumn{3}{|c|}{ Ratawi, Yamama } \\
\hline \multirow[t]{2}{*}{250} & 135 & & & & & \multicolumn{3}{|c|}{ Sulaiy } \\
\hline & 140 & & & & & \multicolumn{3}{|c|}{ Gotnia } \\
\hline
\end{tabular}


Table 4. Calculations and results of thermal maturity values of well KI-1

\begin{tabular}{|c|c|c|c|c|c|c|c|c|}
\hline \multirow{2}{*}{$\begin{array}{c}\text { Depth } \\
(\mathrm{m})\end{array}$} & \multirow{2}{*}{$\begin{array}{l}\text { Time } \\
\text { /Age } \\
\text { (m.y.) }\end{array}$} & \multirow{2}{*}{$\begin{array}{c}\text { Burial rate } \\
\begin{array}{c}\text { dz/dt } \\
(\mathrm{m} . / \mathrm{m} . \mathbf{y} .)\end{array}\end{array}$} & \multirow{2}{*}{$\begin{array}{l}\text { Temp. } \\
\text { grad. } \\
\text { dT/dz } \\
\left({ }^{0} \mathrm{c} / \mathrm{m} .\right)\end{array}$} & \multirow{2}{*}{$\begin{array}{c}\text { Heating } \\
\text { rate }\end{array}$} & \multirow{2}{*}{$\frac{\text { Temp. }}{\left({ }^{0} \mathrm{C}\right)}$} & \multicolumn{3}{|c|}{ Arrhenius equation } \\
\hline & & & & & & $\begin{array}{l}\log _{10} \\
\sum T T I I\end{array}$ & $\begin{array}{c}\mathbf{X} \\
\text { Rem. }\end{array}$ & $\boldsymbol{R}_{\mathrm{o}}$ \\
\hline 0 & 140 & 67.40 & 0.0196 & 1.31 & 20 & & & \\
\hline 337 & 135 & 22.64 & 0.0196 & 0.44 & 27 & -11.16 & & 0.25 \\
\hline 722 & 118 & 57.72 & 0.0196 & 1.13 & 34 & -9.52 & & 0.27 \\
\hline 1357 & 107 & 25.75 & 0.0196 & 0.50 & 47 & -8.34 & & 0.29 \\
\hline 1666 & 95 & 70.20 & 0.0196 & 1.37 & 53 & -7.40 & & 0.32 \\
\hline 2017 & 90 & -64.00 & 0.0196 & -1.25 & 60 & -7.07 & & 0.33 \\
\hline 1953 & 89 & 62.22 & 0.0196 & 1.22 & 58 & -7.07 & & 0.33 \\
\hline 2513 & 80 & 39.50 & 0.0196 & 0.77 & 69 & -6.04 & & 0.38 \\
\hline 2592 & 78 & 50.00 & 0.0196 & 0.98 & 71 & -6.04 & & 0.38 \\
\hline 3092 & 68 & 53.33 & 0.0196 & 1.04 & 81 & -4.82 & & 0.46 \\
\hline 3252 & 65 & -129.66 & 0.0196 & -2.54 & 84 & -4.80 & & 0.46 \\
\hline 2863 & 62 & 96.14 & 0.0196 & 1.88 & 76 & -4.80 & & 0.46 \\
\hline 3536 & 55 & 33.00 & 0.0196 & 0.65 & 89 & -4.31 & & 0.50 \\
\hline 3602 & 53 & 26.58 & 0.0196 & 0.52 & 91 & -4.14 & 1.000 & 0.51 \\
\hline 3921 & 41 & 18.66 & 0.0196 & 0.36 & 97 & -3.20 & 0.999 & 0.62 \\
\hline 4005 & 36.5 & -28.44 & 0.0196 & -0.56 & 98 & -3.20 & 0.999 & 0.62 \\
\hline 3621 & 23 & 27.69 & 0.0196 & 0.54 & 91 & -3.20 & 0.999 & 0.62 \\
\hline 4258 & 0 & & & & 103 & -2.60 & 0.997 & 0.70 \\
\hline \multicolumn{9}{|c|}{ Present-day depth profile to surface } \\
\hline 253 & 0 & & & & & \multicolumn{3}{|c|}{ Dibdiba, Fatha, Ghar } \\
\hline-384 & 23 & & & & & \multicolumn{3}{|c|}{ Unconformity } \\
\hline 84 & 36.5 & & & & & \multicolumn{3}{|c|}{ Dammam, Rus } \\
\hline 319 & 41 & & & & & & & \\
\hline 66 & 53 & & & & & \multicolumn{3}{|c|}{ Umm ER Radhuma } \\
\hline 284 & 55 & & & & & \\
\hline-389 & 62 & & & & & \multicolumn{3}{|c|}{ Unconformity } \\
\hline 160 & 65 & & & & & \multicolumn{3}{|c|}{ Tayarat, Shiranish, Hartha } \\
\hline 500 & 68 & & & & & & & \\
\hline 79 & 78 & & & & & & & \\
\hline 496 & 80 & & & & & \multicolumn{3}{|c|}{ Sadi , Tanuma, Khasib } \\
\hline-64 & 89 & & & & & \multicolumn{3}{|c|}{ Unconformity } \\
\hline 351 & 90 & & & & & \multicolumn{3}{|c|}{ Mishrif, Ruma., Ahm., Mau. } \\
\hline 309 & 95 & & & & & \multicolumn{3}{|c|}{ Nahr Umr } \\
\hline 635 & 107 & & & & & \multicolumn{3}{|c|}{ Shuaiba , Zubair } \\
\hline 385 & 118 & & & & & \multicolumn{3}{|c|}{ Ratawi, Yamama } \\
\hline 337 & 135 & & & & & \multicolumn{3}{|c|}{ Sulaiy } \\
\hline & 140 & & & & & \multicolumn{3}{|c|}{ Gotnia } \\
\hline
\end{tabular}


Table 5. Calculations and results of thermal maturity values of well Ak-1

\begin{tabular}{|c|c|c|c|c|c|c|c|c|}
\hline Depth & $\begin{array}{l}\text { Time } \\
\text { /Age }\end{array}$ & $\begin{array}{c}\text { Burial } \\
\text { rate }\end{array}$ & $\begin{array}{l}\text { Temp. } \\
\text { grad. }\end{array}$ & $\begin{array}{l}\text { Heating } \\
\text { rate }\end{array}$ & Temp. & \multicolumn{3}{|c|}{ Arrhenius equation } \\
\hline (m) & (m.y.) & $\begin{array}{c}\text { dz/dt } \\
(\mathbf{m} . / \mathbf{m} . \mathbf{y} .)\end{array}$ & $\begin{array}{l}\mathrm{dT} / \mathrm{dz} \\
\left({ }^{0} \mathrm{c} / \mathrm{m} .\right)\end{array}$ & $\begin{array}{c}\text { dT/dt } \\
\left({ }^{0} \mathrm{c} / \mathrm{m} . \mathbf{y} .\right)\end{array}$ & $\left({ }^{0} \mathrm{C}\right)$ & $\begin{array}{l}\log _{10} \\
\sum T T I\end{array}$ & $\begin{array}{c}\text { X } \\
\text { Rem. }\end{array}$ & $\boldsymbol{R}_{\boldsymbol{o}}$ \\
\hline 0 & 140 & 21.40 & 0.0205 & 0.44 & 20 & & & \\
\hline 107 & 135 & 25.58 & 0.0205 & 0.52 & 22 & -11.32 & & 0.25 \\
\hline 542 & 118 & 34.18 & 0.0205 & 0.70 & 31 & -9.93 & & 0.26 \\
\hline 918 & 107 & 30.58 & 0.0205 & 0.63 & 39 & -9.09 & & 0.28 \\
\hline 1285 & 95 & 28.80 & 0.0205 & 0.59 & 46 & -8.25 & & 0.29 \\
\hline 1429 & 90 & -15.00 & 0.0205 & -0.31 & 49 & -8.08 & & 0.30 \\
\hline 1414 & 89 & 39.55 & 0.0205 & 0.81 & 49 & -8.08 & & 0.30 \\
\hline 1770 & 80 & 38.33 & 0.0205 & 0.79 & 56 & -7.26 & & 0.32 \\
\hline 1885 & 77 & 45.87 & 0.0205 & 0.94 & 59 & -7.13 & & 0.33 \\
\hline 2252 & 69 & 51.50 & 0.0205 & 1.06 & 66 & -6.29 & & 0.37 \\
\hline 2458 & 65 & -95.00 & 0.0205 & -1.95 & 70 & -6.07 & & 0.38 \\
\hline 2173 & 62 & 113.75 & 0.0205 & 2.33 & 65 & -6.07 & & 0.38 \\
\hline 2628 & 58 & 29.00 & 0.0205 & 0.59 & 74 & -5.86 & & 0.39 \\
\hline 2773 & 53 & 18.00 & 0.0205 & 0.37 & 77 & -5.22 & & 0.43 \\
\hline 3070 & 36.5 & -20.93 & 0.0205 & -0.43 & 83 & -4.28 & & 0.50 \\
\hline 2442 & 6.5 & 1120 & 0.0205 & 2.30 & 70 & -4.28 & & 0.50 \\
\hline 3170 & 0 & & & & & -4.28 & & 0.50 \\
\hline
\end{tabular}

Present-day depth profile to surface

\begin{tabular}{lcc}
100 & 0 & Dibdiba \\
-628 & 6.5 & Unconformity \\
297 & 36.5 & Dammam, Rus \\
145 & 53 & Umm ER Radhuma \\
170 & 58 & \\
-285 & 62 & Unconformity \\
206 & 65 & Tayarat, Shiranish, Hartha \\
367 & 69 & \\
115 & 77 & Sadi , Tanuma, Khasib \\
341 & 80 & Unconformity \\
-15 & 89 & Mishrif, Ruma., Ahm. , Mau. \\
144 & 90 & Nahr Umr \\
367 & 95 & Shuaiba, Zubair \\
376 & 107 & Ratawi, Yamama \\
435 & 118 & Sulaiy \\
107 & 135 & Gotnia \\
\hline
\end{tabular}


Table 6. Calculations and results of thermal maturity values of well Ub-1

\begin{tabular}{|c|c|c|c|c|c|c|c|c|}
\hline \multirow{2}{*}{$\begin{array}{c}\text { Depth } \\
(\mathrm{m})\end{array}$} & \multirow{2}{*}{$\begin{array}{c}\text { Time / } \\
\text { Age }\end{array}$} & \multirow{2}{*}{$\begin{array}{c}\begin{array}{c}\text { Burial } \\
\text { rate }\end{array} \\
\mathbf{d z} / \mathbf{d t} \\
(\mathbf{m} . / \mathbf{m} . \mathbf{y} .)\end{array}$} & \multirow{2}{*}{$\begin{array}{l}\begin{array}{c}\text { Temp. } \\
\text { grad. }\end{array} \\
\text { dT/dz } \\
\left({ }^{0} \mathrm{c} / \mathrm{m} .\right)\end{array}$} & \multirow{2}{*}{$\begin{array}{c}\begin{array}{c}\text { Heating } \\
\text { rate }\end{array} \\
\text { dT/dt } \\
\left({ }^{0} \mathrm{c} / \mathrm{m} . \mathbf{y} .\right)\end{array}$} & \multirow{2}{*}{$\frac{\text { Temp. }}{\left({ }^{0} \mathrm{C}\right)}$} & \multicolumn{3}{|c|}{ Arrhenius equation } \\
\hline & & & & & & $\begin{array}{l} \\
\log _{10} \\
\sum T T I\end{array}$ & \multirow[t]{2}{*}{$\begin{array}{c}\mathrm{X} \\
\text { Rem. }\end{array}$} & $\boldsymbol{R}_{o}$ \\
\hline 0 & 140 & 21.00 & 0.0168 & 0.35 & 20 & & & \\
\hline 105 & 135 & 13.82 & 0.0168 & 0.23 & 22 & -11.23 & & 0.25 \\
\hline 340 & 118 & 35.90 & 0.0168 & 0.60 & 26 & -10.43 & & 0.26 \\
\hline 735 & 107 & 15.50 & 0.0168 & 0.26 & 32 & -9.92 & & 0.26 \\
\hline 921 & 95 & 52.66 & 0.0168 & 0.88 & 35 & -9.34 & & 0.27 \\
\hline 1079 & 92 & 13.00 & 0.0168 & 0.22 & 38 & -9.34 & & 0.27 \\
\hline 1105 & 90 & -13.00 & 0.0168 & -0.22 & 39 & -9.16 & & 0.27 \\
\hline 1092 & 89 & 25.66 & 0.0168 & 0.43 & 38 & -9.16 & & 0.27 \\
\hline 1323 & 80 & 36.87 & 0.0168 & 0.62 & 42 & -8.66 & & 0.28 \\
\hline 1618 & 72 & 41.57 & 0.0168 & 0.70 & 47 & -8.20 & & 0.29 \\
\hline 1909 & 65 & -50.66 & 0.0168 & -0.85 & 52 & -7.69 & & 0.31 \\
\hline 1757 & 62 & 73.66 & 0.0168 & 1.24 & 50 & -7.69 & & 0.31 \\
\hline 2199 & 56 & 34.66 & 0.0168 & 0.58 & 57 & -7.34 & & 0.32 \\
\hline 2303 & 53 & 4.36 & 0.0168 & 0.07 & 59 & -7.12 & & 0.33 \\
\hline 2434 & 23 & 4.56 & 0.0168 & 0.08 & 61 & -6.00 & & 0.38 \\
\hline 2539 & 0 & & & & 63 & -5.85 & & 0.39 \\
\hline \multicolumn{9}{|c|}{ Present-day depth profile to surface } \\
\hline 105 & 0 & & & & & \multicolumn{3}{|c|}{ Dammam, Rus } \\
\hline 131 & 23 & & & & & & & \\
\hline 104 & 53 & & & & & \multicolumn{3}{|c|}{ Umm ER Radhuma } \\
\hline 290 & 56 & & & & & & & \\
\hline-152 & 62 & & & & & \multicolumn{3}{|c|}{ Unconformity } \\
\hline 291 & 65 & & & & & \multicolumn{3}{|c|}{ Tayarat, Shiranish, Hartha } \\
\hline 295 & 72 & & & & & & & \\
\hline 218 & 80 & & & & & \multicolumn{3}{|c|}{ Sadi , Tanuma, Khasib } \\
\hline-13 & 89 & & & & & \multicolumn{3}{|c|}{ Unconformity } \\
\hline 26 & 90 & & & & & \multicolumn{3}{|c|}{ Kifl, Mishrif } \\
\hline 158 & 92 & & & & & \multicolumn{3}{|c|}{ Rumaila, Ahmadi , Maudud } \\
\hline 186 & 95 & & & & & \multicolumn{3}{|c|}{ Nahr Umr } \\
\hline 395 & 107 & & & & & \multicolumn{3}{|c|}{ Shuaiba, Zubair } \\
\hline 235 & 118 & & & & & \multicolumn{3}{|c|}{ Ratawi, Yamama } \\
\hline 105 & 135 & & & & & \multicolumn{3}{|c|}{ Sulaiy } \\
\hline & 140 & & & & & \multicolumn{3}{|c|}{ Gotnia } \\
\hline
\end{tabular}


Table 7. Calculations and results of thermal maturity values of well Sw-1

\begin{tabular}{|c|c|c|c|c|c|c|c|c|}
\hline \multirow{2}{*}{$\begin{array}{l}\text { Depth } \\
\text { (m) }\end{array}$} & \multirow{2}{*}{$\begin{array}{c}\begin{array}{c}\text { Time / } \\
\text { Age }\end{array} \\
\text { (m.y.) }\end{array}$} & \multirow{2}{*}{$\begin{array}{c}\begin{array}{c}\text { Burial } \\
\text { Rate }\end{array} \\
\text { dz/dt } \\
\text { (m./m.y.) }\end{array}$} & \multirow{2}{*}{$\begin{array}{l}\text { Temp. } \\
\text { grad. } \\
\text { dT/dz } \\
\left({ }^{0} \mathrm{c} / \mathrm{m} .\right)\end{array}$} & \multirow{2}{*}{$\begin{array}{c}\begin{array}{c}\text { Heating } \\
\text { rate }\end{array} \\
\text { dT/dt } \\
\left({ }^{0} \mathrm{c} / \mathrm{m} . \mathbf{y} .\right)\end{array}$} & \multirow{2}{*}{$\begin{array}{c}\text { Temp. } \\
\left({ }^{0} \mathrm{C}\right)\end{array}$} & \multicolumn{3}{|c|}{ Arrhenius equation } \\
\hline & & & & & & $\begin{array}{l}\log _{10} \\
\sum T T I\end{array}$ & $\begin{array}{c}X \\
\text { Rem. }\end{array}$ & $R_{o}$ \\
\hline 0 & 140 & 10.60 & 0.022 & 0.23 & 20 & & & \\
\hline 53 & 135 & 9.59 & 0.022 & 0.21 & 21 & -11.42 & & 0.25 \\
\hline 216 & 118 & 30.36 & 0.022 & 0.67 & 25 & -10.43 & & 0.26 \\
\hline 550 & 107 & 13.33 & 0.022 & 0.29 & 32 & -9.94 & & 0.26 \\
\hline 710 & 95 & 61.67 & 0.022 & 1.36 & 36 & -9.19 & & 0.27 \\
\hline 895 & 92 & 3.00 & 0.022 & 0.07 & 40 & -9.19 & & 0.27 \\
\hline 901 & 90 & -3.00 & 0.022 & -0.07 & 40 & -9.19 & & 0.27 \\
\hline 899 & 89 & 26.11 & 0.022 & 0.57 & 40 & -9.19 & & 0.27 \\
\hline 1133 & 80 & 40.72 & 0.022 & 0.90 & 45 & -8.58 & & 0.28 \\
\hline 1581 & 69 & 44.00 & 0.022 & 0.97 & 55 & -7.37 & & 0.32 \\
\hline 1757 & 65 & -30.00 & 0.022 & -0.66 & 59 & -7.14 & & 0.33 \\
\hline 1667 & 62 & 50.66 & 0.022 & 1.11 & 57 & -7.14 & & 0.33 \\
\hline 2123 & 53 & 3.19 & 0.022 & 0.07 & 67 & -6.2 & & 0.37 \\
\hline 2238 & 17 & 3.11 & 0.022 & 0.07 & 69 & -5.19 & & 0.43 \\
\hline 2291 & 0 & & & & 70 & -5.19 & & 0.43 \\
\hline
\end{tabular}

\section{Present-day depth profile to surface}

\begin{tabular}{lcc}
53 & 0 & Dammam, Rus \\
115 & 17 & \\
366 & 53 & Umm ER Radhuma \\
-90 & 62 & Unconformity \\
176 & 65 & Tayarat, Shiranish, Hartha \\
448 & 69 & \\
232 & 80 & Sadi , Tanuma, Khasib \\
-3 & 89 & Unconformity \\
6 & 90 & Kifl \\
185 & 92 & Rumaila, Ahmadi, Maudud \\
160 & 95 & Nahr Umr \\
334 & 107 & Shuaiba, Zubair \\
163 & 118 & Ratawi, Yamama \\
53 & 135 & Sulaiy \\
& 140 & Gotnia \\
\hline
\end{tabular}


Table 8. Calculations and results of thermal maturity values of well Sa-1

\begin{tabular}{|c|c|c|c|c|c|c|c|c|}
\hline \multirow{2}{*}{$\begin{array}{c}\text { Depth } \\
(\mathrm{m})\end{array}$} & \multirow{2}{*}{$\begin{array}{c}\begin{array}{c}\text { Time / } \\
\text { Age }\end{array} \\
\text { (m.y.) }\end{array}$} & \multirow{2}{*}{$\begin{array}{c}\begin{array}{c}\text { Burial } \\
\text { Rate }\end{array} \\
\text { dz/dt } \\
(\mathbf{m} . / \mathbf{m} . \mathbf{y} .)\end{array}$} & \multirow{2}{*}{$\begin{array}{l}\text { Temp. } \\
\text { grad. }\end{array}$} & \multirow{2}{*}{$\begin{array}{c}\begin{array}{c}\text { Heating } \\
\text { rate }\end{array} \\
\text { dT/dt } \\
\left({ }^{0} \mathrm{c} / \mathrm{m} . \mathbf{y} .\right)\end{array}$} & \multirow{2}{*}{$\begin{array}{c}\text { Temp. } \\
\left.{ }^{0} \mathrm{C}\right)\end{array}$} & \multicolumn{3}{|c|}{ Arrhenius equation } \\
\hline & & & & & & $\log _{10} \sum T T I$ & $\begin{array}{c}\mathrm{X} \\
\text { Rem. }\end{array}$ & $\boldsymbol{R}_{o}$ \\
\hline 0 & 140 & 5.60 & 0.0178 & 0.10 & 20 & & & \\
\hline 28 & 135 & 10.11 & 0.0178 & 0.18 & 21 & -11.06 & & 0.25 \\
\hline 200 & 118 & 46.81 & 0.0178 & 0.83 & 24 & -10.56 & & 0.25 \\
\hline 715 & 107 & 15.16 & 0.0178 & 0.27 & 33 & -9.88 & & 0.26 \\
\hline 897 & 95 & 71.33 & 0.0178 & 1.27 & 36 & -9.24 & & 0.27 \\
\hline 1111 & 92 & 18.00 & 0.0178 & 0.32 & 40 & -9.24 & & 0.27 \\
\hline 1147 & 90 & -18.00 & 0.0178 & -0.32 & 40 & -9.24 & & 0.27 \\
\hline 1129 & 89 & 28.66 & 0.0178 & 0.51 & 40 & -9.24 & & 0.27 \\
\hline 1387 & 80 & 36.33 & 0.0178 & 0.64 & 45 & -7.73 & & 0.31 \\
\hline 1714 & 71 & 44.16 & 0.0178 & 0.79 & 51 & -7.73 & & 0.31 \\
\hline 1979 & 65 & -53.33 & 0.0178 & -0.95 & 55 & -7.47 & & 0.32 \\
\hline 1819 & 62 & 66.33 & 0.0178 & 1.18 & 52 & -7.47 & & 0.32 \\
\hline 2416 & 53 & 3.70 & 0.0178 & 0.07 & 63 & -6.63 & & 0.35 \\
\hline 2583 & 8 & 3.50 & 0.0178 & 0.06 & 66 & -5.37 & & 0.42 \\
\hline 2611 & 0 & & & & 67 & -5.37 & & 0.42 \\
\hline \multicolumn{9}{|c|}{ Present-day depth profile to surface } \\
\hline 28 & 0 & & & & & \multicolumn{3}{|c|}{ Dibdiba , Rus } \\
\hline 167 & 8 & & & & & & & \\
\hline 437 & 53 & & & & & \multicolumn{3}{|c|}{ Umm ER Radhuma } \\
\hline-160 & 62 & & & & & \multicolumn{3}{|c|}{ Unconformity } \\
\hline 265 & 65 & & & & & \multicolumn{3}{|c|}{ Tayarat, Shiranish, Hartha } \\
\hline 327 & 71 & & & & & & & \\
\hline 240 & 80 & & & & & \multicolumn{3}{|c|}{ Sadi, Tanuma, Khasib } \\
\hline-18 & 89 & & & & & \multicolumn{3}{|c|}{ Unconformity } \\
\hline 36 & 90 & & & & & \multicolumn{3}{|c|}{ Kifl, Mishrif } \\
\hline 214 & 92 & & & & & \multicolumn{3}{|c|}{ Rumaila, Ahmadi, Maud } \\
\hline 182 & 95 & & & & & \multicolumn{3}{|c|}{ Nahr Umr } \\
\hline 515 & 107 & & & & & \multicolumn{3}{|c|}{ Shuaiba, Zubair } \\
\hline 172 & 118 & & & & & \multicolumn{3}{|c|}{ Ratawi , Yamama } \\
\hline 28 & 135 & & & & & \multicolumn{3}{|c|}{ Sulaiy } \\
\hline & 140 & & & & & \multicolumn{3}{|c|}{ Gotnia } \\
\hline
\end{tabular}


Table 9. Calculations and results of thermal maturity values of well Gh-1

\begin{tabular}{|c|c|c|c|c|c|c|c|c|}
\hline Depth & $\begin{array}{c}\text { Time / } \\
\text { Age }\end{array}$ & $\begin{array}{l}\text { Burial } \\
\text { rate }\end{array}$ & $\begin{array}{l}\text { Temp. } \\
\text { grad. }\end{array}$ & $\begin{array}{l}\text { Heating } \\
\text { rate }\end{array}$ & Temp. & \multicolumn{3}{|c|}{ Arrhenius equation } \\
\hline (m) & (m.y.) & $\begin{array}{c}\mathrm{dz} / \mathrm{dt} \\
(\mathrm{m} . / \mathrm{m} . \mathrm{y} .)\end{array}$ & $\begin{array}{l}\mathrm{dT} / \mathrm{dz} \\
\left({ }^{0} \mathrm{c} / \mathrm{m} .\right)\end{array}$ & $\begin{array}{c}\text { dT/dt } \\
\left({ }^{0} \mathrm{c} / \mathrm{m} . \mathrm{y} .\right)\end{array}$ & $\left({ }^{0} \mathrm{C}\right)$ & $\begin{array}{l}\log _{10} \\
\sum T T I I\end{array}$ & $\begin{array}{c}X \\
\text { Rem. }\end{array}$ & $R_{o}$ \\
\hline 0 & 140 & 2.60 & 0.022 & 0.06 & 20 & & & \\
\hline 13 & 135 & 5.18 & 0.022 & 0.11 & 20 & -11.43 & & 0.25 \\
\hline 101 & 118 & 22.63 & 0.022 & 0.50 & 22 & -10.97 & & 0.25 \\
\hline 350 & 107 & 10.33 & 0.022 & 0.23 & 28 & -10.34 & & 0.26 \\
\hline 474 & 95 & 61.66 & 0.022 & 1.36 & 30 & -10.03 & & 0.26 \\
\hline 659 & 92 & 5.50 & 0.022 & 0.12 & 34 & -10.03 & & 0.26 \\
\hline 670 & 90 & -6.00 & 0.022 & -0.13 & 35 & -9.36 & & 0.27 \\
\hline 664 & 89 & 17.11 & 0.022 & 0.38 & 35 & -9.36 & & 0.27 \\
\hline 818 & 80 & 41.30 & 0.022 & 0.91 & 38 & -9.15 & & 0.27 \\
\hline 1355 & 67 & 31.00 & 0.022 & 0.68 & 50 & -7.90 & & 0.30 \\
\hline 1417 & 65 & -25.33 & 0.022 & -0.56 & 51 & -7.90 & & 0.30 \\
\hline 1341 & 62 & 88.00 & 0.022 & 1.94 & 50 & -7.90 & & 0.30 \\
\hline 1517 & 60 & 25.57 & 0.022 & 0.56 & 53 & -7.90 & & 0.30 \\
\hline 1714 & 53 & 2.17 & 0.022 & 0.05 & 58 & -6.95 & & 0.33 \\
\hline 1816 & 6 & 2.16 & 0.022 & 0.05 & 60 & -5.95 & & 0.38 \\
\hline 1829 & 0 & & & & 60 & -5.95 & & 0.38 \\
\hline \multicolumn{9}{|c|}{ Present-day depth profile to surface } \\
\hline 13 & 0 & & & & & \multicolumn{3}{|c|}{ Dammam } \\
\hline 102 & 6 & & & & & & & \\
\hline 179 & 53 & & & & & \multicolumn{3}{|c|}{ Umm ER Radhuma } \\
\hline 100 & 60 & & & & & & & \\
\hline-76 & 62 & & & & & \multicolumn{3}{|c|}{ Unconformity } \\
\hline 62 & 65 & & & & & \multicolumn{3}{|c|}{ Tayarat, Shiranish, Hartha } \\
\hline 537 & 67 & & & & & & & \\
\hline 148 & 80 & & & & & \multicolumn{3}{|c|}{ Sadi , Tanuma, Khasib } \\
\hline-6 & 89 & & & & & \multicolumn{3}{|c|}{ Unconformity } \\
\hline 11 & 90 & & & & & \multicolumn{3}{|c|}{ Kifl, Mishrif } \\
\hline 185 & 92 & & & & & \multicolumn{3}{|c|}{ Rumaila, Ahmadi, Maud } \\
\hline 124 & 95 & & & & & \multicolumn{3}{|c|}{ Nahr Umr } \\
\hline 249 & 107 & & & & & \multicolumn{3}{|c|}{ Shuaiba, Zubair } \\
\hline 88 & 118 & & & & & \multicolumn{3}{|c|}{ Ratawi, Yamama } \\
\hline 13 & 135 & & & & & \multicolumn{3}{|c|}{ Sulaiy } \\
\hline & 140 & & & & & \multicolumn{3}{|c|}{ Gotnia } \\
\hline
\end{tabular}


Table 10. Calculations and results of thermal maturity values of well $\mathrm{Si}-1$

\begin{tabular}{|c|c|c|c|c|c|c|c|c|}
\hline Depth & $\begin{array}{l}\text { Time/ } \\
\text { Age }\end{array}$ & $\begin{array}{c}\text { Burial } \\
\text { rate }\end{array}$ & $\begin{array}{l}\text { Temp. } \\
\text { grad. }\end{array}$ & $\begin{array}{c}\text { Heating } \\
\text { rate }\end{array}$ & Temp. & \multicolumn{3}{|c|}{ Arrhenius equation } \\
\hline (m) & $(\mathbf{m . y .})$ & $\begin{array}{c}\text { dz/dt } \\
(\mathbf{m} . / \mathrm{m} . \mathbf{y} .)\end{array}$ & $\begin{array}{l}\mathrm{dT} / \mathrm{dz} \\
\left({ }^{0} \mathrm{c} / \mathrm{m} .\right)\end{array}$ & $\begin{array}{c}\text { dT/dt } \\
\left({ }^{0} \mathrm{c} / \mathrm{m} . \mathbf{y} .\right)\end{array}$ & $\left({ }^{0} \mathrm{C}\right)$ & $\begin{array}{l}\log _{10} \\
\sum T T I\end{array}$ & $\begin{array}{c}X \\
\text { Rem. }\end{array}$ & $\boldsymbol{R}_{\boldsymbol{o}}$ \\
\hline 0 & 140 & 3.20 & 0.0179 & 0.06 & 20 & & & \\
\hline 16 & 135 & 9.82 & 0.0179 & 0.18 & 20 & -11.43 & & 0.25 \\
\hline 183 & 118 & 11.91 & 0.0179 & 0.21 & 23 & -10.69 & & 0.25 \\
\hline 314 & 107 & 8.58 & 0.0179 & 0.15 & 26 & -10.37 & & 0.26 \\
\hline 417 & 95 & 41.00 & 0.0179 & 0.73 & 27 & -10.37 & & 0.26 \\
\hline 540 & 92 & 4.50 & 0.0179 & 0.08 & 30 & -10.37 & & 0.26 \\
\hline 549 & 90 & -4.66 & 0.0179 & -0.08 & 30 & -10.37 & & 0.26 \\
\hline 535 & 87 & 23.57 & 0.0179 & 0.42 & 30 & -10.36 & & 0.26 \\
\hline 700 & 80 & 38.82 & 0.0179 & 0.69 & 33 & -9.79 & & 0.26 \\
\hline 1127 & 69 & 38.25 & 0.0179 & 0.68 & 40 & -8.99 & & 0.27 \\
\hline 1280 & 65 & -102.66 & 0.0179 & -1.84 & 43 & -8.81 & & 0.28 \\
\hline 972 & 62 & 18.52 & 0.0179 & 0.33 & 37 & -8.81 & & 0.28 \\
\hline 1361 & 41 & 4.46 & 0.0179 & 0.08 & 44 & -8.76 & & 0.28 \\
\hline 1528 & 4 & 4.00 & 0.0179 & 0.07 & 47 & -7.43 & & 0.32 \\
\hline 1544 & 0 & & & & 48 & -7.43 & & 0.32 \\
\hline
\end{tabular}

Present-day depth profile to surface

\begin{tabular}{ccc}
16 & 0 & Umm ER Radhuma \\
165 & 4 & \\
81 & 41 & \\
-308 & 62 & Unconformity \\
153 & 65 & Tayarat, Hartha \\
427 & 69 & \\
151 & 80 & Sadi, Tanuma \\
-14 & 87 & Unconformity \\
9 & 90 & Mishrif \\
123 & 92 & Rumaila, Ahmadi, Maudud \\
103 & 95 & Nahr Umr \\
131 & 107 & Zubair \\
167 & 118 & Ratawi , Yamama \\
16 & 135 & Sulaiy \\
& 140 & Gotnia \\
\hline
\end{tabular}

\subsection{Volumetric Model of Generated Oil}

This model includes the determination of the proportions for generated and entrapping oil assuming the Sulaiy Formation as the major supplier of oil to most of the Lower Cretaceous reservoirs, Southern Iraq. There are many models proposed to estimate the amount of oil generated in the source rocks as provided by (Goff, 1983), through the following equation: 
Generated Oil $=$ Total volume of generated rock $\times$ Total organic carbon $\times$ Petroleum potential $\times$ Oil part is within hydrocarbons $\times$ Transformation ratio $\times$ Volumetric increase

But these methods and most of the models involve some problems in the application of such models as the difficulty of identifying some of the variables as well as it does not provide a clear way in determining the proportion of migrated oil to generated oil, therefore, reducing the accuracy of these methods. State that all previous studies have not estimated the volume of generated and migrated hydrocarbons from the Sulaiy Formation. Espitalie et al (1988) proposed a new approach in this field based on the geochemical analysis of organic matter. The main framework for applying this model, depends on some of the geochemical analysis derived from Rock- Eval device, which, begin by quantifying the total organic carbon (T.O.C), free hydrocarbons on the rocks $\left(S_{1}\right)$, amount of hydrocarbons generated later by cracking the kerogen with temperature not exceeding $550^{\circ} \mathrm{C}\left(\mathrm{S}_{2}\right)$, transformation ratio $\left(\mathrm{S}_{1} / \mathrm{S}_{1}+\mathrm{S}_{2}\right)$ which takes the symbol (TR) and finally determine the hydrogen index (HI) at different depths. The total generated hydrocarbons at a specified depth $\mathrm{S}_{1} \mathrm{~T}_{\text {(d) }}$ is calculated according to the following equation:

$$
\mathrm{TR}_{(\mathrm{obs})}=\frac{S_{1} T_{(d)}}{S_{1} T_{(d)}+S_{2(d)}}
$$

The computed transformation ratio at recent day and paleo time represent how and where most of the petroleum is generated. These can be changed into area of maturation classes for immature oil and gas generation and over mature region (Hantschel and Kauerauf, 2009). The calculation of the transformation ratio in organic matter TR (obs.) is calculated according to the equation:

$$
\mathrm{TR}_{(\mathrm{obs} .)}=\frac{1200\left(H I_{o}-H I_{d}\right)}{H I_{o}\left(1200-H I_{d}\right)}
$$

The Initial hydrogen index $\left(\mathrm{HI}_{\mathrm{o}}\right)$ derived from the intersection of a tangent of maximum values of (HI) with the earth surface. Based on the above the difference between the total quantity of hydrocarbons generated $\mathrm{S}_{1} \mathrm{~T}_{\text {(d) }}$ and the amount of hydrocarbons remaining in the rock represent the amount of migrated hydrocarbons:

$$
\Delta \mathrm{S}_{1(\mathrm{~d})}=S_{1} T_{(d)}-S_{1(d)}
$$

The migrated hydrocarbons may take a negative signal at some depths. This indicates that the amount of hydrocarbons generated is less than the amount of hydrocarbons remaining in rock, thus, reflecting the low ability of hydrocarbon generation, while, the positive signal that appears at most depths indicates that, the amount of generation is higher than the remained part in rock and in turn indicates a high generation capacity at those depths. In order to estimate migration efficiency, the percentage of migrated hydrocarbons is considered to the total amount of hydrocarbons generated:

$$
\text { Migration efficiency } \%=\frac{100 \cdot \Delta S_{1(d)}}{S_{1} T_{(d)}}
$$

Ungerer (1993) proposed Equations by which to evaluate the initial petroleum efficiency of the source rocks:

$$
\begin{gathered}
\mathrm{TR}_{(\mathrm{d})}=\mathrm{S}_{1(\mathrm{~d})} / \mathrm{S}_{1(\mathrm{~d})}+\mathrm{S}_{2(\mathrm{~d})} \\
\mathrm{TR}_{(\mathrm{d})}=\frac{1200\left(H I_{o}-H I_{d}\right)}{H I_{o}\left(1200-H I_{d}\right)}
\end{gathered}
$$




$$
\begin{gathered}
\mathrm{S}_{2} \mathrm{O}=\left(\mathrm{HI}_{\mathrm{o}} . \mathrm{TOC}_{\mathrm{o}}\right) / 100 \\
\mathrm{PP}_{\mathrm{o}}=\mathrm{S}_{1} \mathrm{~T}_{(\mathrm{d})}+\mathrm{S}_{2} \mathrm{O}_{(\mathrm{d})}
\end{gathered}
$$

Where:

$\mathrm{TR}_{(\mathrm{d})}$ : Calculated transformation ratio at depth (d).

$\mathrm{HI}_{(\mathrm{d})}$ : Hydrogen index at depth (d).

$\mathrm{HI}_{\mathrm{o}}$ : Initial hydrogen index at depth (d).

$\mathrm{S}_{2} \mathrm{O}$ : Initial petroleum potential remaining in organic matter at depth (d).

$\mathrm{TOC}_{\mathrm{o}}$ : Initial total organic carbon at depth (d).

$\mathrm{PP}_{\mathrm{o}}$ : Initial petroleum potential at depth (d).

By using these equations, results showed that the main generation zone within the Sulaiy Formation is limited to depths (4480 - 4510 m.) (Fig. 9).

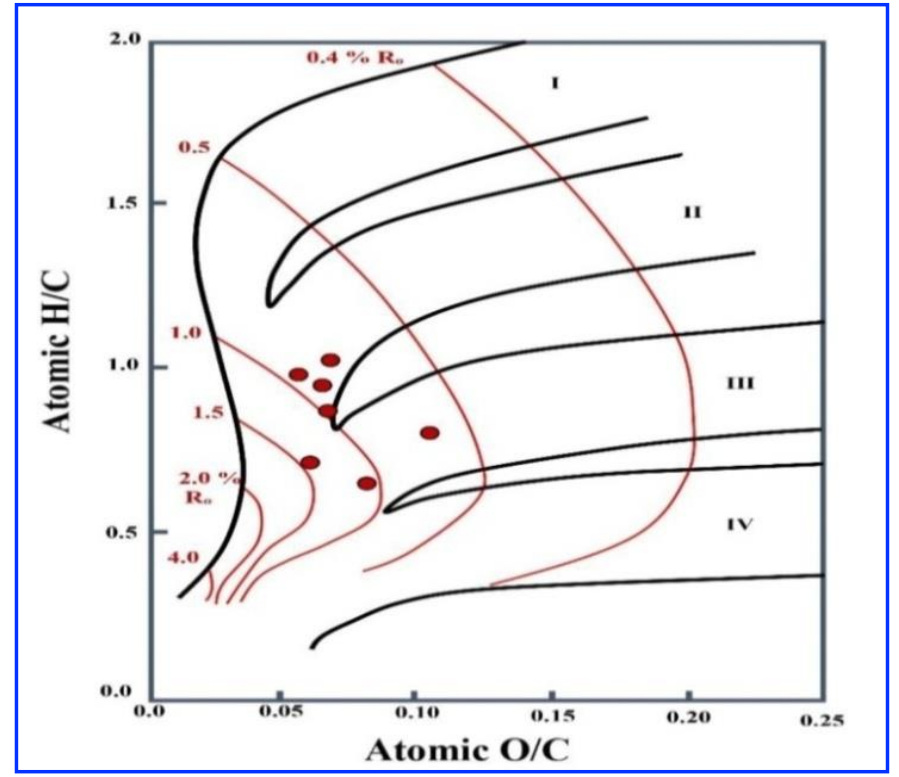

Fig.4. Van Krevelen plot showing kerogen type of the Sulaiy Formation

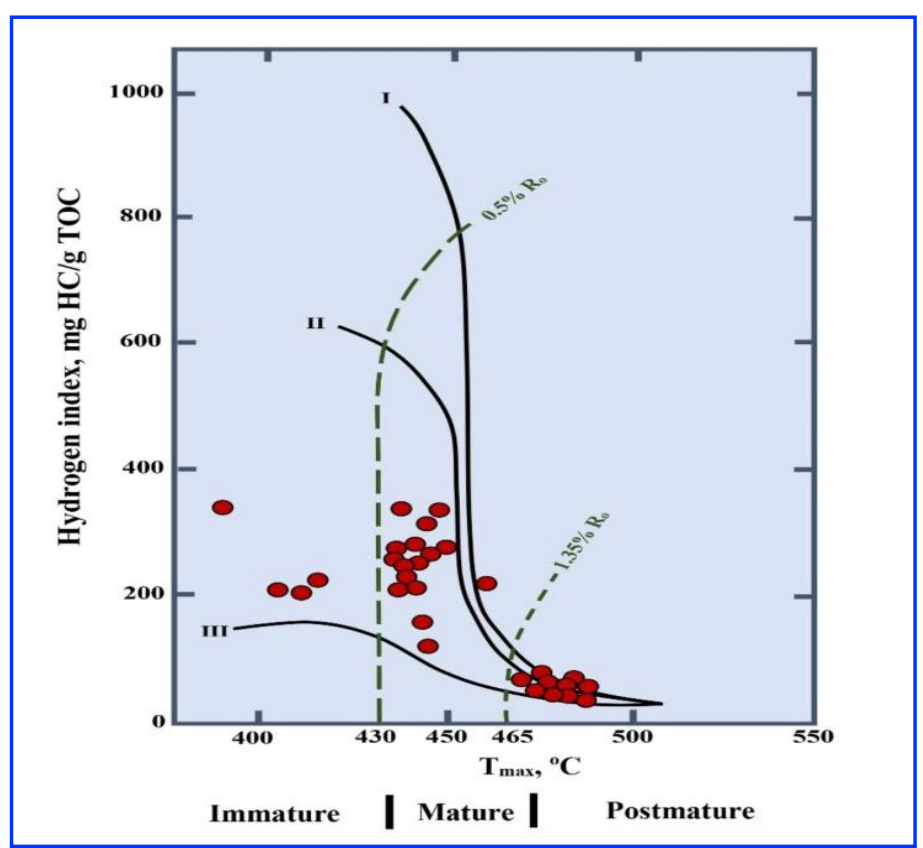

Fig.5 A plot of HIo versus Tmax for the Sulaiy Formation 


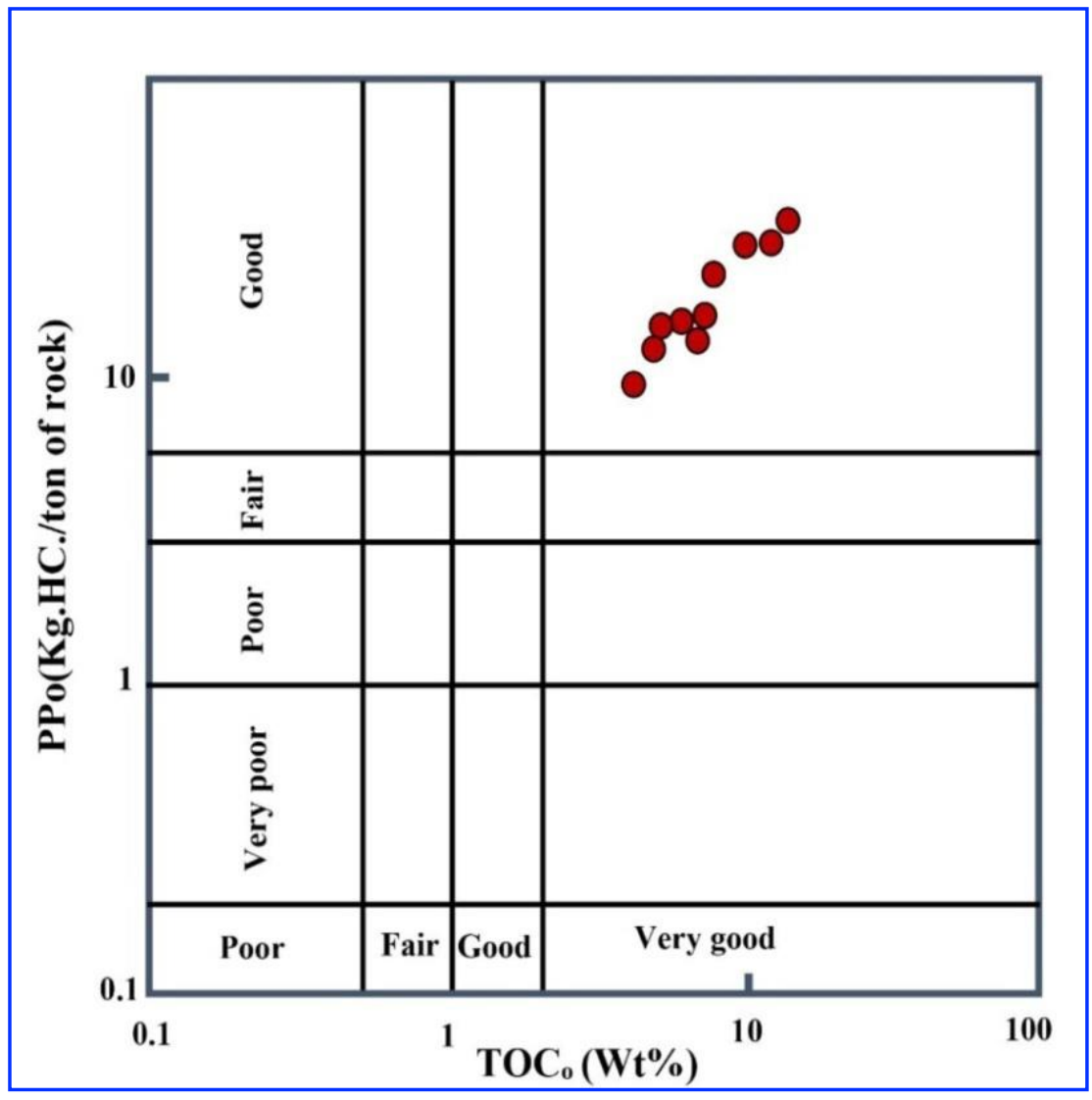

Fig.6. A plot of PPo versus T.O.C.o for the lower part of the Sulaiy Formation

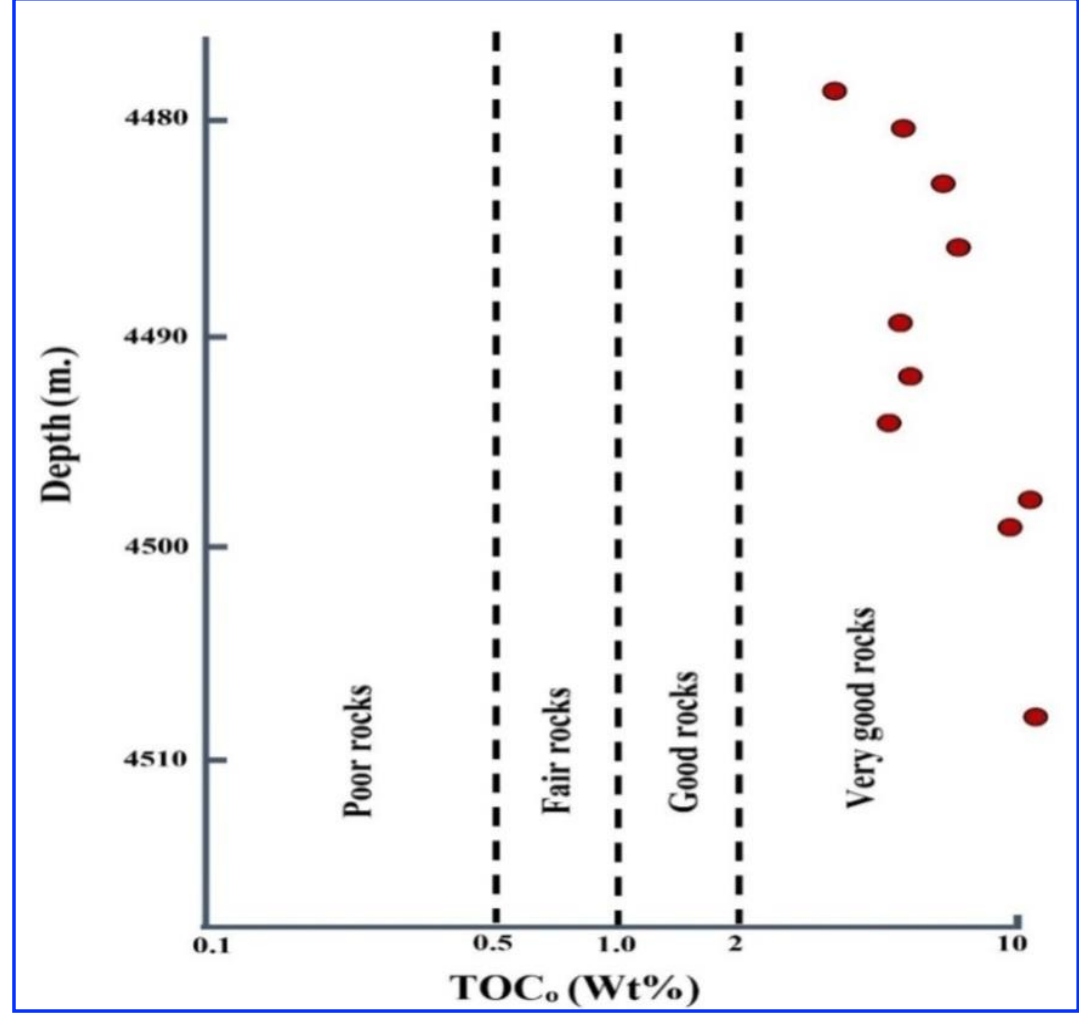

Fig.7. A plot of T.O.C.o versus depth for the lower part of the Sulaiy Formation 


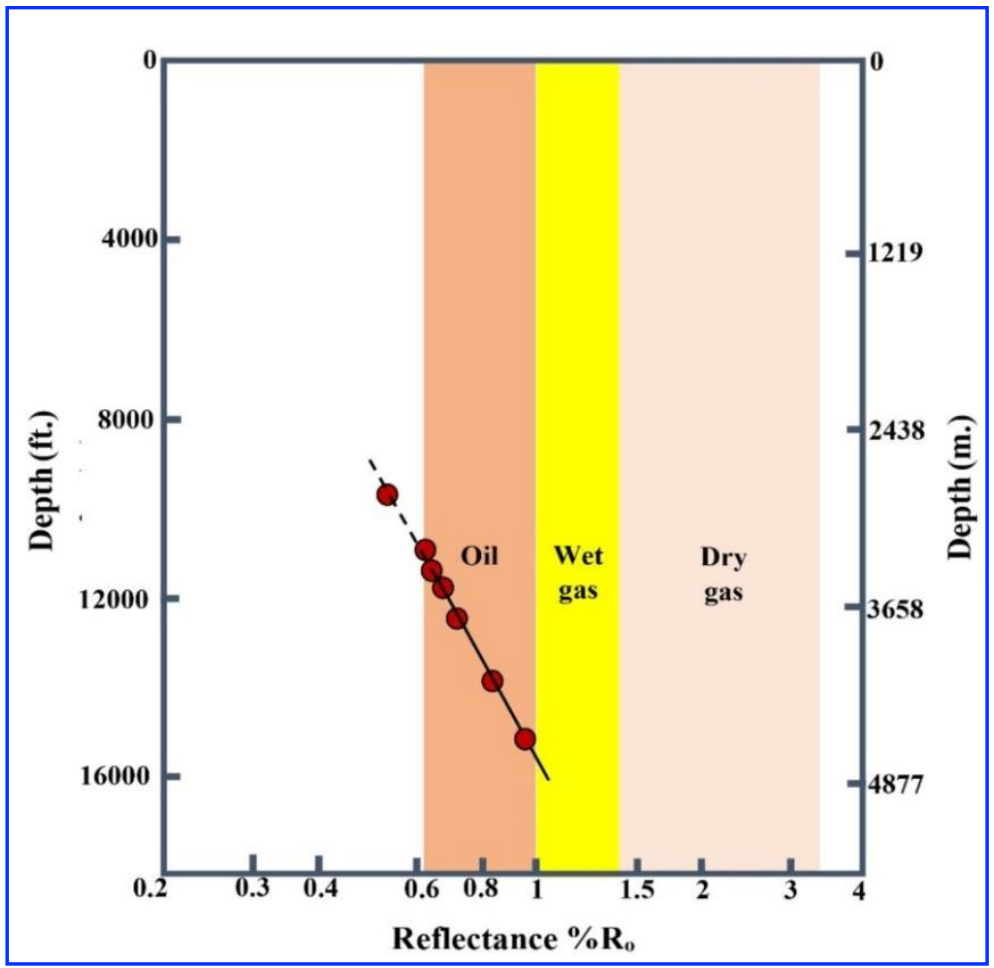

Fig. 8. Estimation of the maturation zone for the Sulaiy Formation

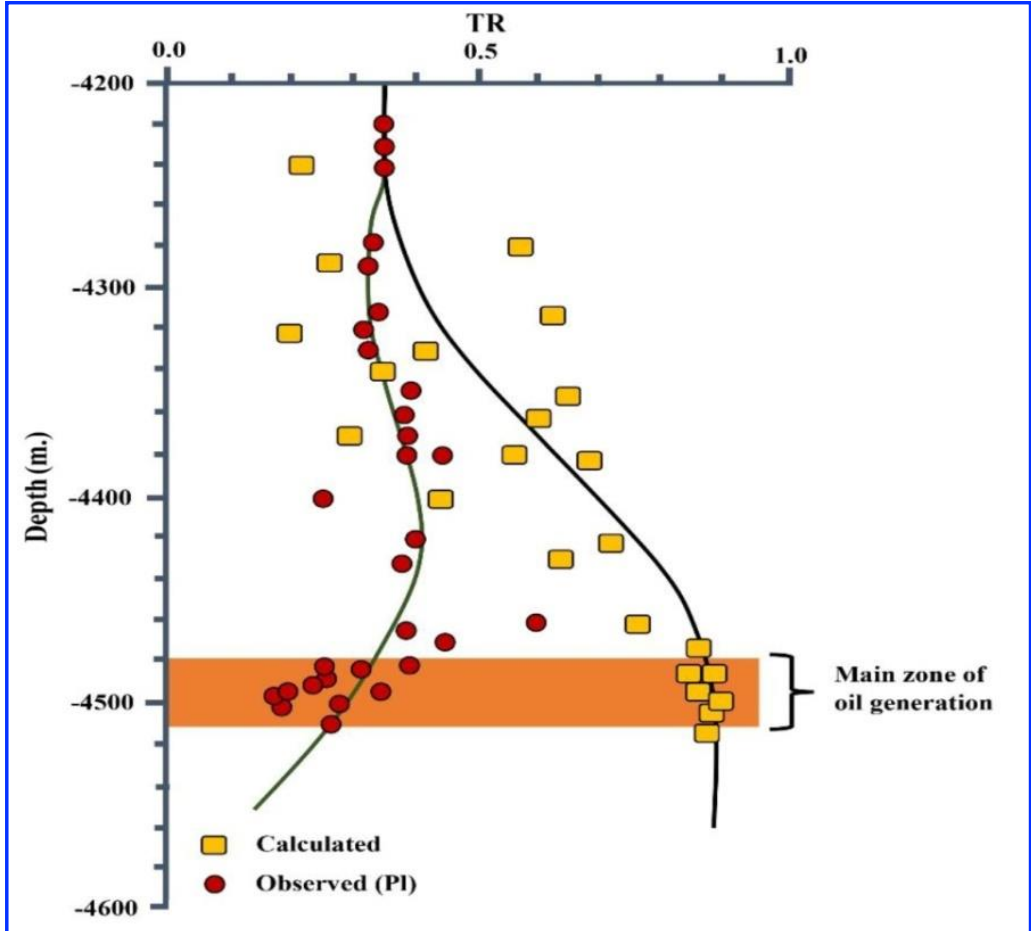

Fig.9. PI and TR calculated-depth relationship is indicated by the main zone of oil generation

\subsection{Estimated Volume of Generated Oil}

The calculation below acquires great important as they enable us to answer many questions such as: determine the depth of onset of migration, amount of generated and migrated hydrocarbons, as well as, the efficiency and potential migration trends. The most studies in this field indicated that the extracting in the Sulaiy Formation is higher than in all Formations. In Zb-42 that start 10.35 Ma by 0.055 
$\mathrm{M}$ tons $/ \mathrm{km}^{2}$ and end at $0.22 \mathrm{Ma}$ by $1.118 \mathrm{M}$ tons $/ \mathrm{km}^{2}$ (Handhal, et al. 2020). it is possible to determine the mature area of the Sulaiy Formation. Through our prior knowledge of the generated thickness, the volume of the generated rock is calculated:

Total rock weight $(\mathrm{gm})=$. total rock volume $\left(\mathrm{cm}^{3}\right) \times$ rock density $\left(\mathrm{gm} . / \mathrm{cm}^{3}\right)$

$$
=\left(1928 \times 10^{15}\right) \times(2.45)=4897 \times 10^{15} \mathrm{gm} \text {. }
$$

This is where the average rate of the initial organic matter (TOC) within this part of the Sulaiy Formation calculated in the present study is equal to $7.8997 \%$. The total weight of the organic matter convertible to hydrocarbons is $386.81 \times 10^{15} \mathrm{gm}$ (Fig. 9). According to the above geochemical equations the rate of migratory hydrocarbons within this zone is equal to $14.90 \mathrm{mg} / \mathrm{gm}$. Consequently, the weight of the organic matter convertible to migratory hydrocarbons amounts to $5763.47 \times 10^{15} \mathrm{mg}$. To convert this weight into a volume unit, the density of migrated oil must be determined. The density of oil in most of the Lower Cretaceous reservoir in southern Iraq is about ( $\left.35^{\circ} \mathrm{API}\right)$ which is equivalent to the density of $0.85 \mathrm{gm} / \mathrm{cm}^{3}$. Thus, the amount of migrated hydrocarbons is equivalent to $6.78 \times 10^{9} \mathrm{~m}^{3}$ or $42.6 \times 10^{9}$ bbl. This amount of migrated oil, which was estimated in this study according to Arrhenius equation, exceeds that estimated in Al-Kubaisi (1994) that depended on the Lopatin method or Time- Temperature Index, which estimated the migrated oil at $19.8 \times 10^{9} \mathrm{bbl}$. The reason for this, as mentioned above, is due to the under estimation of Lopatin method at high heating rates. This amount of migrated oil may exceed the amount of oil trapped, as a result of the lost oil during the stages of migration. However, it represents a good amount for supply the Lower Cretaceous reservoirs of southern Iraq and some of the adjacent areas with late vertical migration. Here, one must emphasize the need to assume early lateral migration originated from mature units eastward to Iran supplied oil to most of the Middle Cretaceous reservoirs in southern Iraq and northern Kuwait.

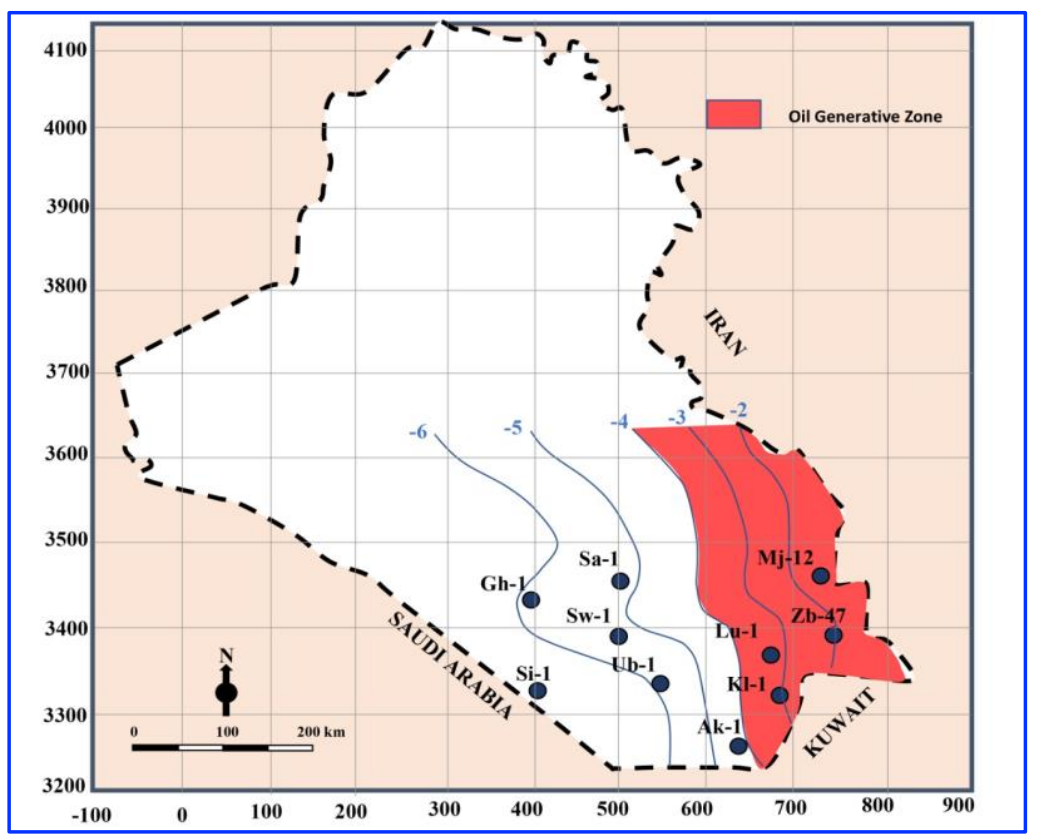

Fig.10. Iso -TTI map showing the thermal maturation zone of the sulaiy Formation

\section{Conclusions}

The adaption of thermal maturation model based on Arrhenius equation is of great important, by determining the change in the degree of thermal maturation horizontally and vertically for units that could be the rocks or part of them may have generated oil. In particular, the values of the determined indices showed a clear correlation with the results of geochemical analyzes. This model also showed the 
real rates of thermal maturation level of the Sulaiy Formation compared with understanding imation he showed by Lopatin model (AL-Kubaisi,1994). It should be noted that dealing with source rock in advanced stages of maturity requires a special evaluation that differs from dealing with immature rocks. The latter represents the original potentiality of the rocks, while the mature rocks that lost some of their generative capacity during the stages of maturity. Accordingly, what is measured now represents the remaining potential in the rock and not the initial capacity, this is an important aspect that was not covered by most previous research and studies. According to the values of the TTI calculated in this study, the map of Iso-thermal maturation (Iso-TTI) of the Sulaiy Formation indicated a significant increase in thermal maturation values eastward due to high burial rates and geothermal gradient towards the center of the basin. The TTI values in the south eastern part of Iraq, specifically at the study area, indicates a significant increase in thermal maturation rates compared to central and western Iraq (AlAtroshi, 1989). The result of the study indicated that the entry of the Sulaiy Formation rocks within the thermal maturation zone was at 52 million years ago, at the end of Lower Eocene in addition, the lower parts of the formation are characterized by a high content of organic matter which is mostly marine. It has a high potential to produce liquid hydrocarbons was estimated about $\left(42.6 \times 10^{9}\right.$ bbl. $)$ so it can be considered that the formation rocks are good source of reservoir in southern Iraq and some neighboring areas. The high thermal maturation rates eastward is accompanied by an increase in the content of organic matter which becomes more marine towards the center of basin. This provides mature source rocks and high capacity for oil generation which is a good source of early lateral migration to the reservoirs of southern Iraq.

\section{Acknowledgements}

The authors are very grateful to the Editor in Chief Prof. Dr. Salih M. Awadh, the Secretary of Journal Mr. Samir R. Hijab. and the Technical Editors for their great efforts and valuable comments.

\section{References}

Al-Ameri, T. K., Al-Musawi, F. S., 2011. Hydrocarbon generation potential of the uppermost Jurassic -basal Cretaceous Sulaiy Formation, South Iraq. Arabian Journal Geoscience, 4 (4), 53-58.

Al-Atroshi, M. A., 1989. Generation, Migration and Accumulation of Hydrocarbons in East Baghdad oil field, M.Sc. Thesis, University of Saladeen, 176p.

Al-Kubaisi, B. F., 1994. Source-Reservoir-Seal Relationships, in Zubair Field's Oil Entrapment, Southern Iraq, M.Sc. Thesis, University of Baghdad, 106p.

Allen, P. A., Allen, J. R., 2005. Basin analysis. Blackwell publishing, second edition, 549 pp.

Al-Mimar, H. S., 2014. Petroleum System Analysis and Reservoir Development of AD' Daima Oil Field, Messan Governorate, South Iraq. M.Sc. Thesis, College of Science, Baghdad University, 115 p.

Al- Zaidi, M. D. A., 2019. Total Petroleum System of Dhi Qar Oil Fields, South of Iraq. Ph. D. Thesis, College of Science, University of Baghdad, $209 \mathrm{p}$.

Baskin, D. K., 1997. Atomic H/C ratio of kerogen as an estimate of thermal maturity and organic matter conversion. American Association of Petroleum Geologists, 81, 1437-1450.

Connan, J., 1974. Time-temperature relation in oil genesis, American Association of Petroleum Geologists, 58, 2516-2521.

Ejedawe, J. E., and Coker, S. J. L., Coker. 1984. Dynamic interpretation of organic matter maturation and evolution of oil generative window: American Association of Petroleum Geologists, 68, 1024-1028.

Ejedawe, J. E., D. O. lambert - Aikhionbare, D. O., K. B. Alofe, K. B., and F. O. Adoh, F.O., 1984. Evolution of the oil generative window and oil and gas occurrences in the Teriary Niger delta basin, American Association of Petroleum Geologists, 68) 11), 1744-1751.

Espitalié, J., Maxwell, J.R., Chenet, Y. and Marquis, F., 1988. Aspects of hydrocarbon migration in the Mesozoic in the Paris Basin as deduced from an organic geochemical survey. In Organic Geochemistry in Petroleum Exploration ( 467-481). Pergamon. 
Goff, J. C., 1983. Hydrocarbon generation and migration from Jurassic source rocks in the E Shetland Basin and Viking Graben of the northern North Sea. Journal of the Geological Society, 140(3), 445-474.

Gorbachev, V. M., 1975. A solution of the exponential integral in the non-iso thermal kinetics for linear heating. Journal of Thermal Analysis, 8 (2), 349-350.

Handhal, A. M., Mahdi, M. M., 2016. Basin modeling analysis and organic maturation for selected wells from deferent fields, Southern Iraq. Modeling Earth System and Environment, 2 (4) ,189.

Hatif, M., Al-Shahwan, M. F., Al-Yaseri, A., 2020. Comparative organic geochemical study of Cretaceous reservoirs of Zubair oilfield, southern Iraq. Iraqi Geological Journal, 53 (2D), 128-147.

Handhal, A. M., Al-sharhan, M., Chafeet, H. A., 2020. Interpretation of hydrocarbon generation, migration, and thermal history of Mesopotamian basin Southern Iraq based 1D Petromod software; Iraqi Geological Journal, 53, (1B), 29-56.

Hantschel, T., Kauerauf, A. I., 2009. Fundamentals of basin and petroleum system modeling, Springer, 476 pp.

Ibrahim, M. W. 1983. Petroleum Geology of Southern Iraq. American Association of Petroleum Geologists. 67, 97-130.

Ibrahim, M. W. 1984. Geothermal gradients and geothermal oil generation in southern Iraq: a preliminary investigation, Journal Petroleum Geology, 7 (1), 77-86.

Japan national oil corporation, 1988. Integrated geological interpretation of the South western area of Iraq (table \& figures): Tokyo, Japan.

Jassim, S. Z., Buday, T., 2006. Late Tithonian-Early Tuonian Megasequence Ap 8. In: Jassim, S. Z. and Goff, J. C. (eds)., 2006; Geology of Iraq. Published by Dolin, Prrague and Moravian Museum, Berno, 341pp.

Karweil, J., 1956. Die metamorphose der kohlen vom standpunkt der physikalischen chemie. Zeitschrift der deutschen geologischen Gesellschaft, 132-139.

Learche, I. R. F., Yarzab, C. G., Kendall, S. C., 1984. Determination of paleo heat flux from vitrinite reflectance. American Association of Petroleum Geologists, 68, 1704-1717.

Lewan, M. D., 1985. Evaluation of petroleum generation by hydrous pyrolysis experimentation: Philosophical Transactions of the Royal Society of London, Series A, 315, 123-134.

Lopatin, N. V. 1971. Temperature and geologic time as factors in coalification: Akademiya Nauk SSSR Izvestiya, seriya geologic heskaya, 3, 95-106 (English translation by Bostick, Illinois state geological survey, 1972).

Mamaseni, W. J., 2020. Petroleum potentiality and petrophysical evaluation of the Middle-Jurassic Sargelu Formation, Northern Iraq. Iraqi Geological Journal, 53 (2D): 77-93.

Oil Exploration Company, 1987. Geochemical study for south eastern of Iraq (Unpubl. Report).

Royden, L., Sclater, J.G. and Herzen, R.V., 1980. Continental margin subsidence and heat flow: important parameters in formation of petroleum hydrocarbons. American Association of Petroleum Geologists, 64(2), 173-187.

Snowdon, L.R., 1979. Errors in Extrapolation of Experimental Kinetic Parameters to Organic Geochemical Systems: GEOLOGIC NOTES. American Association of Petroleum Geologists Bulletin, 63(7), 1128-1134.

Tissot, B.P., 1984. Recent advances in petroleum geochemistry applied to hydrocarbon exploration. American Association of Petroleum Geologists Bulletin, 68(5), 545-563.

Tissot, B. and Espitalie, J., 1975. L'evolution thermique de la matière organique des sédiments: applications d'une simulation mathématique. Potentiel pétrolier des bassins sédimentaires de reconstitution de l'histoire thermique des sédiments. Revue de l'Institut Francais du Petrole, 30(5), 743-778.

Tissot, B., D. H. Welte, D.H., 1978. Petroleum Formation and Occurrence: New York, Springer-Verlag, 538p.

Ungerer, P.F., 1993. Modeling of petroleum generation and expulsion - Un update to recent reviews, in: Basin modeling: Advances and applications, (ed. A. G. Dore et al.): NPF Spec. Publ. 3, 219 - 232.

Waples, D.W., 1980. Time and temperature in petroleum formation: application of Lopatin's method to petroleum exploration. American Association of Petroleum Geologists bulletin, 64(6), 916-926.

Waples, D. W., 1982. Organic geochemistry for exploration geologists: Minneapolis, Burgess Publ. Co. 151p.

Wood, D.A., 1988. Relationships between thermal maturity indices calculated using Arrhenius equation and Lopatin method: implications for petroleum exploration. AAPG bulletin, 72(2),115-134. 